\title{
A SIMPLE METHOD FOR COMPRESSIBLE MULTIFLUID FLOWS*
}

\author{
RICHARD SAUREL ${ }^{\dagger}$ AND RÉMI ABGRALL $\ddagger$
}

\begin{abstract}
A simple second order accurate and fully Eulerian numerical method is presented for the simulation of multifluid compressible flows, governed by the stiffened gas equation of state, in hydrodynamic regime. Our numerical method relies on a second order Godunov-type scheme, with approximate Riemann solver for the resolution of conservation equations, and a set of nonconservative equations. It is valid for all mesh points and allows the resolution of interfaces. This method works for an arbitrary number of interfaces, for breakup and coalescence. It allows very high density ratios (up to 1000). It is able to compute very strong shock waves (pressure ratio up to $10^{5}$ ). Contrary to all existing schemes (which consider the interface as a discontinuity) the method considers the interface as a numerical diffusion zone as contact discontinuities are computed in compressible single phase flows, but the variables describing the mixture zone are computed consistently with the density, momentum and energy. Several test problems are presented in one, two, and three dimensions. This method allows, for example, the computation of the interaction of a shock wave propagating in a liquid with a gas cylinder, as well as Richtmeyer-Meshkov instabilities, or hypervelocity impact, with realistic initial conditions. We illustrate our method with the Rusanov flux. However, the same principle can be applied to a more general class of schemes.
\end{abstract}

Key words. compressible multicomponents flows, compressible multifluid flows, Godunov schemes, nonconservative systems

AMS subject classifications. 65M06, 65M99, 76T05

PII. S1064827597323749

1. Introduction. The direct numerical simulation of compressible multiphase flows is an important research topic with various key applications, ranging from the dynamics of bubbles in nuclear flows to hypervelocity impact or shock wave interaction with densities and material discontinuities: Richtmeyer-Meshkov instabilities, bubble deformation and collapse, gas mixing for combustion, etc. The main applications are in fluid mechanics, material science, astrophysics, and nuclear science.

Here we consider multifluid flows with an hydrodynamic behavior only. Dissipative effects are neglected but they should be included in the model without major difficulties. Under this assumption, the fundamental equations are the Euler equations.

The resolution of the Euler equations in single phase flows is now achieved with reasonable accuracy by many numerical methods. Over the last two decades, the resolution of such flows has become a routine matter with Godunov [10] and high order schemes $[35,30]$. Although this is true for single phase flows, the resolution of similar flows involving fluid interfaces between two gases or two fluids of different physical nature is much more difficult. Interface problems with two gases occur in many combustion applications and are not solved correctly with classical numerical methods $[1,20,33]$. The difficulty lies in the differences between the equation of state parameters for each fluid. This difficulty appears more dramatically with interfaces separating gases and liquid or gases and solid.

Under very high pressures, liquids and solids become compressible. The hydro-

${ }^{*}$ Received by the editors June 27, 1997; accepted for publication (in revised form) May 27, 1998; published electronically December 3, 1999.

http://www.siam.org/journals/sisc/21-3/32374.html

${ }^{\dagger}$ IUSTI, CNRS UMR 6595, 5 rue Enrico Fermi, 13453 Marseille Cedex 13, France.

${ }^{\ddagger}$ Université Bordeaux I, 351 cours de la Libération, 33405 Talence, France (abgrall @ math. u-bordeaux.fr). 
dynamic part of the stress tensor becomes predominant compared with its deviatoric part. The Euler equations become a valid model for such flows. Under high pressures, liquid and solids behave like gases.

It is possible to describe these materials under high pressure with reasonable accuracy by using the stiffened gas equation of state $[17,11]$. Its algebraic form is similar to the ideal gas equation of state (EOS). Each phase of flows involving various materials may be described by an EOS of this type. Only the coefficients of the EOS will vary discontinuously from one medium to another across the interface. This discontinuous variation of these coefficients is at the heart of the difficulty for pressure evaluation at the interface. Any classical fluid dynamics method produces an artificial diffusion of density and internal energy at the interfaces. Since the density ratio between each fluid may be very large, typically of the order of $10^{3}$ for problems we consider here, the numerical values of the density inside the diffusion zone make pressure evaluation by the gas or the liquid EOS very difficult. In general, such Eulerian methods fail at the second time step. This is why specific algorithms have been developed over the last 40 years - to eliminate numerical diffusion at the interfaces. A short review of these methods will be given in the next section.

These methods are all complicated. Some of them are simple conceptually but are difficult to code. We propose here a new method which considers the problem from a different point of view. Numerical diffusion is tolerated in this method and the same algorithm is used at each mesh point-liquid, solid, or gas - and at all interfaces. Its programming is very simple compared with others methods. Its execution time on a computer is not longer than for a single fluid flow calculation. The essential limitation is related to the EOS. Currently, the method applies only to the stiffened gas EOS.

The basic idea of the method is due to Abgrall [2], who considers two gases having a different ratio of specific heats, separated by an interface, to be flowing in a onedimensional (1D) shock tube. This method is generalized to the stiffened gas EOS and hence to multifluid problems, then to multidimensional problems.

As shown in the last section of the paper, this method is suitable for various problems, such as two-phase shock tube, Richtmeyer-Meshkov instabilities, shockbubble interaction, and hypervelocity impact.

2. A review of existing methods. The literature provides references to several families of methods suitable for interface problems and compressible flows. Each of these methods considers different ways of eliminating numerical diffusion at the interfaces.

2.1. Lagrangian methods. The most classical and natural way to compute flows with interfaces is to use Lagrangian methods: in this framework the mesh moves with the flow. The interfaces are characterized by specific positions in the flow and move with the local velocity. If the method does not use artificial viscosity, the interface will remain sharp. In general it is necessary to use some artificial viscosity in the shock waves, and the interfaces smear during time when shock waves interact with them. Specific algorithms based on "master and slave" surfaces for nodes at the interface restore a sharp profile. However, since large displacements are current in hydrodynamic flows, the mesh suffers of large distortions. These distortions induce errors on the solution and it is necessary to rezone the mesh periodically. Moreover, fluid dynamics applications deal frequently with fluid inflows and outflows. In the context of Lagrangian methods this implies addition and elimination of meshes, yielding an extra complexity. The mesh management produces inaccuracies and difficulties in the coding. An excellent review of these methods is given in Benson [3]. 
2.2. Front tracking methods. Front tracking methods use a classical Eulerian solver for points away from the interface and a specific scheme for the points around the interface. Some examples of the capabilities of such methods may be found in Young, Glimm, and Boston [38]. Each point of the interface is propagated at each time step in a spatially operator-split fashion, using a local coordinate system aligned with the interface. The tangential split steps are based on finite differences in a plane tangent to the interface. The normal propagation step is based on a Riemann solver, which is an exact solution of the local $1 \mathrm{D}$ conservation law with a discontinuity. A nonlocal correction to this purely local resolution of the dynamics of the discontinuity at the interface is provided by a finite difference computation based on the method of characteristics. See [9] for further details. Harten and Hyman [15] have proposed a conservative front tracking scheme, relying on a self-adjusting grid procedure around the interface. This scheme is very efficient in one dimension but difficult to extend in two dimensions. A conservative two-dimensional (2D) scheme is proposed in Leveque and Shyue [23] and a nonconservative one is proposed in Mao [24]. This last one relies on variable extrapolations through the interface. The basic idea of this scheme is simple, but its $2 \mathrm{D}$ coding is difficult, like for the others methods. Another conceptually simple method was proposed by Cocchi and Saurel [4]. Starting from a sharp interface (as initial data, for example), an Eulerian scheme will produce a nonphysical numerical diffusion on the two points only around the interface during a single time step. An exact Riemann solver consists of an exact solution of the Euler equation at a given location. The two points suffering from numerical diffusion may be corrected using the states given by the exact Riemann solver. The variables at other Eulerian nodes are not affected by numerical diffusion. A linear interpolation between the Riemann solution and the Eulerian solution, on the same side of the interface, is sufficient to restore a sharp discontinuity. The 1D variant of this method is very simple and efficient [5]. Its extension has been realized in two dimensions [4], but the method becomes rather complex. This is the main drawback of these methods. Indeed, these schemes are in general very efficient and accurate, but even when the fundamental idea of the scheme is simple, its coding is difficult. These schemes involve dynamic distribution of markers along the interface, the localization of neighboring marker points in the Eulerian mesh, and the managing of interface geometrical singularities like triple points. Each of these steps render these methods very difficult to extend in three dimensions and to problems involving interface breakup or coalescence. Despite these drawbacks, the accuracy of such methods is generally very good, and considerable efforts have been produced by specialized research teams to extend these methods in three dimensions and for breakup and coalescence interface problems (Glimm et al. [9]).

2.3. Eulerian methods. Eulerian methods are not really Eulerian since they are in general a combination of Lagrange and projection methods. Also, they use specific schemes at the interfaces. They are based on the volume of fluid (VOF) idea [18] and reconstruction methods [39]. These methods are widely used in hydrocodes and seem to be very efficient. They are based on advection algorithms for interface reconstruction. Knowing the volume fraction and the velocity field of each fluid at one time step, they are able to predict the volume fraction and the interface location at the next time step.

The knowledge of the interface position is sufficient for incompressible flows to determine the density field. For a compressible flow, the determination of the densities and internal energy of each fluid in a mixed cell is not obvious at all. Papers in the literature comment very little on this point. The only paper the authors have found 
is the one by Norman and Winkler [27], which describes a complicated (but efficient) method for the determination of densities and internal energies. This procedure is based on a relaxation method for each fluid variable. The densities and internal energies are iterated and compared with the mixture ones once pressure equilibrium is reached. In fact, the literature is too poor to evaluate these methods. However, since the applications are very important, specialized teams have developed such codes. The results obtained by these schemes seem to agree well with experiments.

More recently, a new type of Eulerian method has been proposed. These methods are based on a localization of the interface by the level set method [26, 20]. Karni's method [20] switches the energy formulation equation from conservative to primitive variables in order to prevent pressure oscillations at the interface. Comparison with the technique of Mulder, Osher, and Sethian [26] shows large accuracy improvement by this switching procedure. Extension of these methods to reactive flows and fluids governed by the stiffened gas equation is not obvious. The difficulty comes from the large variations of the EOS parameters at the interface, and in particular from the corrective pressure $\pi$ in (3.1). The method we are presenting in the following works correctly with the stiffened gas equation and can be extended to reactive flows.

2.4. Arbitrary Lagrangian-Eulerian methods. Arbitrary Lagrangian-Eulerian (ALE) methods are Lagrangian at the interfaces and use moving grid strategies with Eulerian schemes away from the interfaces. The difficulty lies in managing the mesh when several interfaces are present in the flow.

To summarize, when there is only a small number of interfaces and low flow velocities, Lagrangian methods are efficient. When the flow velocity increases, mesh distortion increases, too, and ALE methods are recommended. Front tracking methods are the most accurate. They are also the most difficult to code, to generalize to an arbitrary number of interfaces and three-dimensional (3D) problems. Eulerian methods allow an arbitrary number of interfaces and large fluid velocities but little information on these methods is available in the open literature.

These considerations have led us to conduct investigations on simpler methods for flows at high velocities, with an arbitrary number of interfaces and strong shock waves. The main drawback of our method is that it was restricted, until now, to materials governed by the stiffened gas EOS.

3. The method. The algorithm follows the basic ideas of Abgrall [2] for the resolution of the 1D Euler equations with ideal gas EOS for interfaces between two gases of different ratios of specific heats. In the following we extend this method in multidimensions for fluids governed by the stiffened gas EOS.

3.1. The stiffened gas EOS. The stiffened gas EOS $[11,17]$ is written

$$
p=(\gamma-1) \rho I-\gamma \pi,
$$

where $p$ denotes the pressure, $\rho$ the density, and $I$ the internal energy. The constants $\gamma$ and $\pi$ are parameters characteristic of the material. This expression is identical to the ideal gas EOS when $\gamma$ represents the ratio of specific heats and $\pi$ is zero. Equation (3.1) is a reasonable approximation for gases, liquid, and solid under high pressure conditions.

The parameters $\gamma$ and $\pi$ are determined by adjusting the experimental and theoretical Hugoniot curves. For a broad class of materials, the experimental Hugoniot curve can be represented as a linear function:

$$
U_{s}=c_{0}+s U_{p},
$$




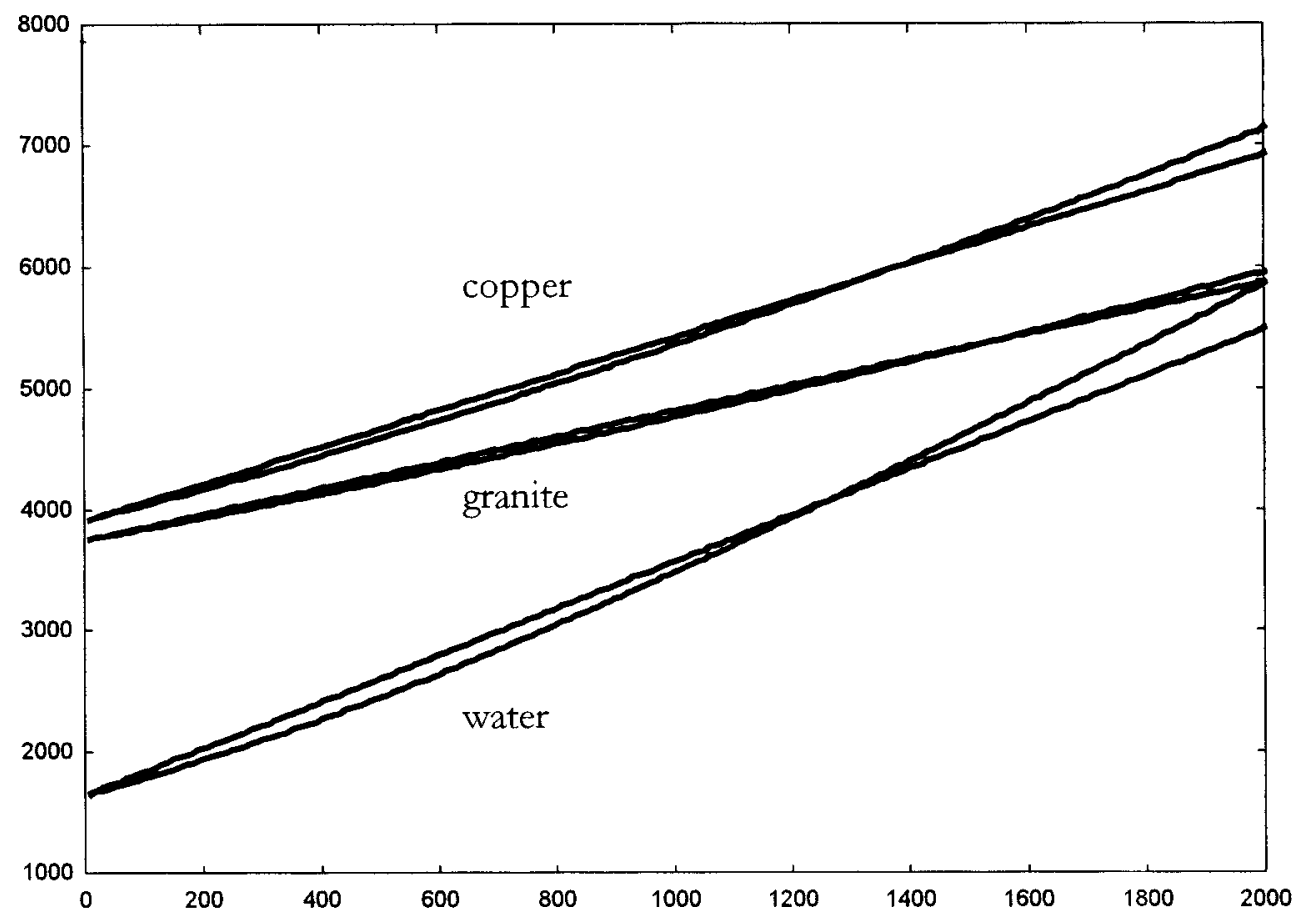

FIG. 1. Experimental and theoretical Hugoniot curves for granite, water, and copper. The straight lines are the experimental ones.

where $c_{0}$ is the sound speed in the material at rest, $U_{s}$ is the shock wave velocity, $U_{p}$ is the material velocity, and $s$ a dimensionless constant. The Rankine-Hugoniot relations may be combined with (3.1) (see Cocchi, Saurel, and Loraud [5]):

$$
U_{s}=\left[c_{0}^{2}+\left(\frac{\gamma+1}{4} U_{p}\right)^{2}\right]^{1 / 2}+\frac{\gamma+1}{4} U_{p} .
$$

By choosing an interval of variation of the fluid velocity, one can determine the value of $\gamma$ giving the closest agreement between the experimental curve and the model EOS. Once the coefficient $\gamma$ is determined, the value of $\pi$ is obtained from

$$
\pi=\frac{\rho_{0} c_{0}^{2}}{\gamma}-P_{0},
$$

where $\rho_{0}$ represents the material initial density and $P_{0}$ the ambient pressure.

Figure 1 represents the theoretical and experimental curves for liquid water (lower curve), copper (upper curve), and granite. The experimental curves are obtained from data given in Marsh [25].

The stiffened gas EOS parameter may always be adjusted in order to get a close agreement with experimental curves (linear curves). The corresponding parameters are summarized in Table 3.1. The simulations of section 4 are done with this set of parameters.

3.2. Basic version of the method. To illustrate our method, we choose the simplest ingredients of a conservative first order Godunov-type scheme in one dimen- 
TABLE 3.1

Some parameters of the stiffened gas EOS for a model gas, liquid, and solids.

\begin{tabular}{|c||cccc|}
\hline & Air & Water & Copper & Granite \\
\hline Density $\left(\mathrm{kg} / \mathrm{m}^{3}\right)$ & 1 & 998 & 8924 & 2627 \\
$c_{0}(\mathrm{~m} / \mathrm{s})$ & 340 & 1647 & 3910 & 3750 \\
$s$ & - & 1.921 & 1.51 & 1.06 \\
$\gamma$ & 1.4 & 4.4 & 4 & 2.6 \\
$\pi(\mathrm{Pa})$ & 0 & $6.10^{8}$ & $34110^{8}$ & $14210^{8}$ \\
\hline
\end{tabular}

sion. It will be generalized to different schemes, second order accurate, and several space dimensions in the next section.

The basic idea behind our method is that any physical contact discontinuity should remain a contact discontinuity. More precisely, we demand that in any flow where the pressure and the velocity are uniform, since these properties are respected in the exact solution whatever the physical characteristic of the flow, then the pressure and the velocity will remain uniform whatever the time.

This property may not be true, even for the simplest example of multifluid flow as shown in $[1,20]$. This is a consequence of the following fact: The numerical dissipation associated with an exact conserved variable may not be consistent with the property "p and u" uniform.

We ask for the flow solver to respect this physical property. This property is in fact in contradiction with the strict respect of conservation as it was clearly understood by Karni [20]. By considering a single supersonic contact discontinuity, it can be seen that this wrong behavior is not solver dependant but is a general property of any upwind scheme (see $[1,6]$ ). We have to abandon the strict-conservativity. This may be done in several ways (Karni [21]) and we want to loosen this property as little as possible.

The simplest way to achieve this goal is first to enforce mass, momentum, and energy conservation. The physical description of the flow (for example its composition) is described by another equation which is discretized in such a way that the contact discontinuities are respected. In order to achieve this goal as simply as possible, the basic flow solver needs only to fulfill

$$
\text { if } u_{i}^{(1)}=u \text { and } P_{i}^{(1)}=P \text { for any } i \text {, then } u_{i}^{(2)}=u \text { and } P_{i}^{(2)}=P,
$$

where superscripts (1) and (2) denote two consecutive time steps.

We illustrate these principles on the examples of the Rusanov flux [31] with the stiffened gas EOS. In another section, we show how to generalize our methodology to several space dimension and different numerical fluxes.

3.3. Modification of the Godunov-Rusanov scheme. The equations to solve in each fluid and at the interface are the Euler equations written in conservative form:

$$
\frac{\partial U}{\partial t}+\frac{\partial F}{\partial x}=0
$$

where $U=(\rho, \rho u, \rho e)^{T}$ and $F=\left(\rho u, \rho u^{2}+P, u(\rho e+P)\right)^{T}$, and $\rho, u$, and $P$ are, respectively, density, velocity, and pressure. The total energy is defined by $e=I+\frac{u^{2}}{2}$. The pressure and the internal energy are related through (3.1), where $\gamma$ and $\pi$ depend on the material. 
The system (3.5) has to be supplemented by two equations which provide evolution of the phases:

$$
\begin{aligned}
& \frac{\partial \gamma}{\partial t}+u \frac{\partial \gamma}{\partial x}=0 \\
& \frac{\partial \pi}{\partial t}+u \frac{\partial \pi}{\partial x}=0
\end{aligned}
$$

What we intend to do is to provide a discretization of (3.6) which will ensure a correct treatment of the pressure.

We consider a mesh of constant spacing $\Delta x$. The solution is evolved with a time step $\Delta t$. The first order Godunov scheme reads

$$
U_{i}^{n+1}=U_{i}^{n}-\frac{\Delta t}{\Delta x}\left(F_{i+1 / 2}^{n}-F_{i-1 / 2}^{n}\right),
$$

where the numerical flux is given by [31]

$$
F_{i+1 / 2}=F\left(U_{i}, U_{i+1}\right):=\frac{1}{2}\left(F_{i}+F_{i+1}-S\left(U_{i+1}-U_{i}\right)\right),
$$

where $S=\max \left\{\left|u_{i+1}+a_{i+1}\right|,\left|u_{i+1}-a_{i+1}\right|,\left|u_{i}+a_{i}\right|,\left|u_{i}-a_{i}\right|\right\}$ and $a$ is the speed of sound $a=\sqrt{\gamma \frac{P+\pi}{\rho}}$.

Now we assume that $u$ and $P$ are uniform in the flow. The density $\rho \gamma, \pi$ may not be uniform. More specifically at time $t_{n}$, we assume $u_{i}=u, P_{i}=P$ for any $i$, whereas $\gamma_{i}=\gamma_{l}, \pi_{i}=\pi_{l}$ if $i<0$ and $\gamma_{i}=\gamma_{r}, \pi_{i}=\pi_{r}$ if $i \geq 0$. We note $\lambda=\frac{\Delta t}{2 \Delta x}$. We get

$$
\begin{aligned}
\rho_{i}^{n+1}=\rho_{i}^{n}-\lambda\left[u_{i}^{n}\left(\rho_{i+1}^{n}-\rho_{i}^{n}\right)-S_{i+1 / 2}\left(\rho_{i+1}^{n}-\rho_{i}^{n}\right)\right. & \left.+S_{i+1 / 2}\left(\rho_{i}^{n}-\rho_{i-1}^{n}\right)\right], \\
\rho_{i}^{n+1} u_{i}^{n+1}=\rho_{i}^{n} u_{i}^{n}-\lambda\left[\left(\left(\rho u^{2}+P\right)_{i+1}^{n}-\left(\rho u^{2}+P\right)_{i-1}^{n}\right)\right. & +S_{i+1 / 2}\left(\rho_{i+1}^{n} u_{i+1}^{n}-\rho_{i}^{n} u_{i}^{n}\right) \\
& \left.+S_{i+1 / 2}\left(\rho_{i}^{n} u_{i}^{n}-\rho_{i-1}^{n} u_{i-1}^{n}\right)\right] .
\end{aligned}
$$

Combining (3.9) and (3.10), the equality of pressure and velocity at time $t_{n}$, one gets

$$
u_{i}^{n+1}=u \text {. }
$$

Now we consider the equation for the total energy:

$$
\begin{aligned}
\rho e_{i}^{n+1}=\rho e_{i}^{n}-\lambda\left[(\rho e u+P u)_{i+1}^{n}-(\rho e u+P u)_{i}^{n}\right. & -S_{i+1 / 2}\left(\rho e_{i+1}^{n}-\rho e_{i}^{n}\right) \\
& \left.+S_{i-1 / 2}\left(\rho e_{i}^{n}-\rho e_{i+1}^{n}\right)\right] .
\end{aligned}
$$

Using (3.9), (3.11), and pressure uniformity, we get

$$
\rho I_{i}^{n+1}=\rho I_{i}^{n}-\lambda\left[u_{i}^{n}\left(\rho I_{i+1}^{n}-\rho I_{i-1}^{n}\right)-S_{i+1 / 2}\left(\rho I_{i+1}^{n}-\rho I_{i}^{n}\right)+S_{i-1 / 2}\left(\rho I_{i}^{n}-\rho I_{i-1}^{n}\right)\right] .
$$

With the help of (3.1), with $\rho I=\frac{P+\gamma \pi}{\gamma-1}:=\alpha P+\beta$ with $\alpha=\frac{1}{\gamma-1}$ and $\beta=\frac{\gamma \pi}{\gamma-1}$, we get

$$
\begin{aligned}
(\alpha P)_{i}^{n+1}+\beta_{i}^{n+1} & =(\alpha P)_{i}^{n}-\lambda\left[u_{i}^{n} P_{i}^{n}\left(\alpha_{i+1}^{n}-\alpha_{i-1}^{n}\right)-S_{i+1 / 2} P_{i}^{n}\left(\alpha_{i+1}^{n}-\alpha_{i}^{n}\right)\right. \\
& \left.+S_{i-1 / 2} P_{i}^{n}\left(\alpha_{i}^{n}-\alpha_{i-1}^{n}\right)\right] \\
& +\beta_{i}^{n}-\lambda\left[u_{i}^{n}\left(\beta_{i+1}^{n}-\beta_{i-1}^{n}\right)-S_{i+1 / 2}\left(\beta_{i+1}^{n}-\beta_{i}^{n}\right)+S_{i-1 / 2}\left(\beta_{i}^{n}-\beta_{i-1}^{n}\right)\right] .
\end{aligned}
$$


Hence, we obtain $P_{i}^{n+1}=P_{i}^{n}$ under the condition

(3.12) $\chi_{i}^{n+1}=\chi_{i}^{n}-\lambda\left[u_{i}^{n}\left(\chi_{i-1}^{n}-\chi_{i-1}^{n}\right)-S_{i+1 / 2}\left(\chi_{i+1}^{n}-\chi_{i}^{n}\right)+S_{i-1 / 2}\left(\chi_{i}^{n}-\chi_{i-1}^{n}\right)\right]$, where $\chi=(\alpha, \beta)$.

To summarize, the scheme in the general case is

$$
\begin{aligned}
& U_{i}^{n+1}=U_{i}^{n}-\lambda\left(F_{i+1 / 2}^{n}-F_{i-1 / 2}^{n}\right), \\
& \chi_{i}^{n+1}=\chi_{i}^{n}-\lambda\left[u_{i}^{n}\left(\chi_{i-1}^{n}-\chi_{i-1}^{n}\right)-S_{i+1 / 2}\left(\chi_{i+1}^{n}-\chi_{i}^{n}\right)+S_{i-1 / 2}\left(\chi_{i}^{n}-\chi_{i-1}^{n}\right)\right] .
\end{aligned}
$$

The last equation is a numerical discretization of

$$
\frac{\partial \chi}{\partial t}+u \frac{\partial \chi}{\partial x}=0
$$

which can be seen to be equivalent to the system (3.6).

3.4. Extension to second order. The extension is realized by following the Monotonic Upstream Schemes for Conservative Laws (MUSCL)-Hancock method (see Quirk [28]). The flow variables are characterized by a mean value $U_{i}^{n}$ and a slope $\delta U_{i}^{n}$.

For the sake of simplicity and efficiency, the slopes are calculated on primitive variables, $W=(\rho, u, P)^{T}$, since we are interested essentially on the accuracy of the scheme at the interface. At the interface, slopes of $u$ and $P$ are zero. The primitive variables on the cell boundary are given by

$$
\begin{aligned}
& W_{i+1 / 2,-}^{n}=W_{i}^{n}+\frac{1}{2} \delta W_{i}^{n}, \\
& W_{i-1 / 2,+}^{n}=W_{i}^{n}-\frac{1}{2} \delta W_{i}^{n} .
\end{aligned}
$$

Various components of primitive vector $W$ are then obtained easily:

$$
\begin{aligned}
& \rho_{i+1 / 2,-}^{n}=\rho_{i}^{n}+\frac{1}{2} \delta \rho_{i}^{n}, \\
& \rho_{i-1 / 2,+}^{n}=\rho_{i}^{n}-\frac{1}{2} \delta \rho_{i}^{n}, \\
& u_{i+1 / 2,-}^{n}=u_{i-1 / 2,+}^{n}=u, \\
& P_{i+1 / 2,-}^{n}=P_{i-1 / 2,+}^{n}=P .
\end{aligned}
$$

This satisfies automatically the contact discontinuity conditions. The mean conservative variable vector at time $t_{n+1 / 2}$ may be calculated by

$$
U_{i}^{n+1}=U_{i}^{n}-\frac{\lambda}{2}\left[F\left(U_{i+1 / 2,-}^{n}-F\left(U_{i-1 / 2,+}^{n}\right)\right] .\right.
$$

Use of (3.15) in (3.16) yields the result

$$
\begin{aligned}
\rho_{i}^{n+1 / 2} & =\rho_{i}^{n}-\frac{\lambda}{2} u_{i}^{n} \delta \rho_{i}^{n}, \\
u_{i}^{n+1 / 2} & =u_{i}^{n}=u, \\
\chi_{i}^{n+1 / 2} & =\chi_{i}^{n}-\frac{\lambda}{2} \delta \chi_{i}^{n} .
\end{aligned}
$$

The predictor step of the MUSCL procedure ends with the calculation of the cell boundary variables at time $t_{n+1 / 2}$,

$$
W_{i+1 / 2, \pm}^{n+1 / 2}=W_{i}^{n+1 / 2} \mp \frac{1}{2} \delta W_{i}^{n}
$$

and the same for the $\chi$ vector.

The predictor step of the MUSCL scheme may be summarized as follows: 
1. Compute the primitive variables and the slopes at time $t_{n}$.

2. Compute the cell boundary variables with (3.15).

3. Update at time $t_{n+1 / 2}$ the conservative variables by (3.16) and the nonconservative one by (3.17).

4. Compute the cell boundary variables at time $t_{n+1 / 2}$ by (3.18). The corrector step now is written

$$
U_{i}^{n+1}=U_{i}^{n}-\lambda\left[F\left(U_{i+1 / 2,-}^{n+1 / 2}, U_{i+1 / 2,+}^{n+1 / 2}\right)-F\left(U_{i-1 / 2,-}^{n+1 / 2}, U_{i-1 / 2,+}^{n+1 / 2}\right)\right] .
$$

Using the Rusanov flux (3.7) in (3.19) and the same principles as in the preceding section, one gets the discretization of $\chi$ ensuring pressure and velocity equality at the interface:

$$
\begin{aligned}
\chi_{i}^{n+1}=\chi_{i}^{n} & -\lambda\left[u_{i}^{n+1 / 2}\left(\chi_{i+1 / 2,-}^{n+1 / 2}+\chi_{i+1 / 2,+}^{n+1 / 2}-\chi_{i-1 / 2,-}^{n+1 / 2}-\chi_{i-1 / 2,+}^{n+1 / 2}\right)\right. \\
& \left.-S_{i+1 / 2}^{n+1 / 2}\left(\chi_{i+1 / 2,+}^{n+1 / 2}-\chi_{i+1 / 2,-}^{n+1 / 2}\right)+S_{i-1 / 2}^{n+1 / 2}\left(\chi_{i-1 / 2,+}^{n+1 / 2}-\chi_{i-1 / 2,-}^{n+1 / 2}\right)\right] .
\end{aligned}
$$

Conservative variables are updated by (3.19) while nonconservative one are updated by $(3.20)$.

3.5. Extension to other schemes. As we have pointed out before, the numerical schemes are not all suitable for our modification. In order to simplify the calculation, it is necessary that the following condition be true: If the pressure and the velocity are uniform on the stencil of the numerical scheme, then the velocity stays uniform at $t_{n+1}$. For example, the van Leer [35] fluxes do not satisfy the above constraint.

The basic idea is to update the conservative variables by the original numerical scheme and to impose further conditions on the energy equation so that the pressure will remain uniform. In [2], the Roe scheme has been modified along these lines. In the next paragraph, we show how to modify the Harten, Lax, and van Leer (HLL) scheme [16].

3.5.1. Extension with the HLL approximate Riemann solver. The HLLRiemann solver assumes an estimate of the right and left wave speed $S^{+}$and $S^{-}$at a cell boundary. The numerical flux in the HLL approximation is written

$$
F_{H L L}=\frac{S^{+} F_{L}-S^{-} F_{R}+S^{+} S^{-}\left(U_{R}-U_{L}\right)}{S^{+}-S^{-}} .
$$

The subscripts $R$ and $L$ stand for the right and left states and fluxes at the cell boundary. Various choices are possible for the wave speed estimates; see for example [34]. Here we use Davis estimates [7]:

$$
S^{+}=\max \left(0, u_{L}+a_{L}, u_{R}+a_{R}\right), S^{-}=\min \left(0, u_{L}-a_{L}, u_{R}-a_{R}\right) .
$$

The first order development of the method as given in section 3.1 with the HLL solver yields the following discretization of the nonconservative equations (3.14):

$$
\begin{aligned}
\chi_{i}^{n+1}=\chi_{i}^{n}-\lambda\left[\frac{u_{i}^{n}\left(S_{i+1 / 2}^{+} \chi_{i}^{n}-S_{i+1 / 2}^{-} \chi_{i+1}^{n}\right)+S_{i+1 / 2}^{+} S_{i+1 / 2}^{-}\left(\chi_{i+1}^{n}-\chi_{i}^{n}\right)}{S_{i+1 / 2}^{+}-S_{i+1 / 2}^{-}}\right. \\
\left.-\frac{u_{i}^{n}\left(S_{i-1 / 2}^{+} \chi_{i}^{n}-S_{i-1 / 2}^{-} \chi_{i}^{n}\right)+S_{i-1 / 2}^{+} S_{i-1 / 2}^{-}\left(\chi_{i}^{n}-\chi_{i-1}^{n}\right)}{S_{i-1 / 2}^{+}-S_{i-1 / 2}^{-}}\right] .
\end{aligned}
$$


For the extension to second order, relations given in section 3.3 remain valid except that relation (3.20) must be replaced by

$$
\begin{aligned}
& \chi_{i}^{n+1}=\chi_{i}^{n}-\lambda \\
& \times\left[\frac{u_{i}^{n+1 / 2}\left(S_{i+1 / 2}^{+} \chi_{i+1 / 2,-}^{n+1 / 2}-S_{i+1 / 2}^{-} \chi_{i+1 / 2,+}^{n+1 / 2}\right)+S_{i+1 / 2}^{+} S_{i+1 / 2}^{-}\left(\chi_{i+1 / 2,+}^{n+1 / 2}-\chi_{i+1 / 2,+}^{n+1 / 2}\right)}{S_{i+1 / 2}^{+}-S_{i+1 / 2}^{-}}\right. \\
& \left.-\frac{u_{i}^{n+1 / 2}\left(S_{i-1 / 2}^{+} \chi_{i-1 / 2,-}^{n+1 / 2}-S_{i-1 / 2}^{-} \chi_{i-1 / 2,+}^{n+1 / 2}\right)+S_{i-1 / 2}^{+} S_{i-1 / 2}^{-}\left(\chi_{i-1 / 2,+}^{n+1 / 2}-\chi_{i-1 / 2,-}^{n+1 / 2}\right)}{S_{i-1 / 2}^{+}-S_{i-1 / 2}^{-}}\right],
\end{aligned}
$$

where $\lambda=\frac{\Delta t}{\Delta x}$.

We now show how to modify the Roe scheme [30].

3.5.2. Extension with the Roe approximate Riemann solver. To design a numerical scheme relying on the Roe fluxes, we follow the same approach as in [2]. We begin by assuming that an averaged Jacobian matrix is available for the following system of PDEs. It describes the evolution of a fluid made of two components.

$$
\begin{aligned}
& \frac{\partial \rho_{1} Y_{1}}{\partial t}+\frac{\partial \rho_{1} u Y_{1}}{\partial x}=0, \\
& \frac{\partial \rho_{2} Y_{2}}{\partial t}+\frac{\partial \rho_{2} u Y_{2}}{\partial x}=0, \\
& \frac{\partial \rho u}{\partial t}+\frac{\partial\left(\rho u^{2}+P\right)}{\partial x}=0, \\
& \frac{\partial E}{\partial t}+\frac{\partial u(E+P)}{\partial x}=0 .
\end{aligned}
$$

Here, $\rho_{1}$ and $\rho_{2}$ are the densities of each fluid and $Y_{1}, Y_{2}$ are the respective volume fraction ( $Y_{i}=$ volume occupied by fluid $i /$ total volume). The total energy per unit volume is given by $E=\rho e$. The mixture pressure is given by $P=Y_{1} P_{1}+Y_{2} P_{2}$, where $P_{1}$ and $P_{2}$ are given by the corresponding stiffened gas EOS. Since pressure equilibrium between the two fluids is assumed, the mixture of state is written

$$
I=\frac{P}{\gamma-1}+\frac{\gamma \pi}{\gamma-1}
$$

where

$$
\frac{1}{\gamma-1}=\frac{Y_{1}}{\gamma_{1}-1}+\frac{Y_{2}}{\gamma_{2}-1} \quad \text { and } \quad \frac{\gamma \pi}{\gamma-1}=\frac{Y_{1} \gamma_{1} \pi_{1}}{\gamma_{1}-1}+\frac{Y_{2} \gamma_{2} \pi_{2}}{\gamma_{2}-1} .
$$

We denote by $\bar{A}$ the averaged Jacobian matrix and we assume it admits the following structure:

$$
\bar{A}=\left(\begin{array}{cccc}
\bar{u} \overline{Y_{1}} & -\bar{u} \overline{Y_{1}} & \overline{Y_{1}} & 0 \\
-\bar{u} \bar{Y}_{2} & \bar{u} \overline{Y_{2}} & \overline{Y_{2}} & 0 \\
-\bar{u}^{2}+\widetilde{P}_{\rho_{1} Y_{1}} & -\bar{u}^{2}+\widetilde{P}_{\rho_{2} Y_{2}} & 2 \bar{u}+\widetilde{P}_{m} & \widetilde{P}_{E} \\
\bar{u}\left(-\bar{H}+\widetilde{P}_{\rho_{1} Y_{1}}\right) & \bar{u}\left(-\bar{H}+\widetilde{P}_{\rho_{2} Y_{2}}\right) & \bar{H}+\bar{u} \widetilde{P}_{m} & \bar{u}\left(1+\widetilde{P}_{E}\right)
\end{array}\right) .
$$


In (3.26), $\bar{u}, \bar{H}, \overline{Y_{i}}$ are the standard Roe averages of the velocity $u$, the total enthalpy per unit volume $H$, and the volume fractions $Y_{i}$, respectively, while $\widetilde{P}_{\rho_{1} Y_{1}}$, $\widetilde{P}_{\rho_{2} Y_{2}}, \widetilde{P}_{m}$, and $\widetilde{P}_{E}$ are approximations of the partial derivatives $P_{\rho_{1} Y_{1}}, P_{\rho_{2} Y_{2}}, P_{m}$, and $P_{R}(m=\rho u)$. In the appendix, we give the precise expressions of these quantities as well as a way to get them. What is important is that $\bar{A}$ admit the following set of eigenvectors:

$$
r_{1}=\left(\begin{array}{c}
1 \\
0 \\
\bar{u} \\
\frac{\bar{u}^{2}}{2}-\frac{\xi_{1}}{\kappa}
\end{array}\right), r_{2}=\left(\begin{array}{c}
0 \\
1 \\
\bar{u} \\
\frac{\bar{u}^{2}}{2}-\frac{\xi_{2}}{\kappa}
\end{array}\right), r_{3}=\left(\begin{array}{c}
Y_{1} \\
Y_{2} \\
\bar{u}-\bar{c} \\
\bar{H}-\bar{u} \bar{c}
\end{array}\right), r_{4}=\left(\begin{array}{c}
Y_{1} \\
Y_{2} \\
\bar{u}+\bar{c} \\
\bar{H}+\bar{u} \bar{c}
\end{array}\right),
$$

where $\kappa=P_{E}, \xi_{i}=P_{\rho_{i} Y_{i}}-K \frac{u^{2}}{2}$, and $c$ is the speed of sound. The vectors $r_{1}$ and $r_{2}$ are associated to the eigenvalue $u$.

Assume that $u$ and $P$ are uniform on the mesh. Take two neighboring cells $i$ and $i+1$. Since

$$
F\left(U_{i+1}\right)-F\left(U_{i}\right)=u\left(U_{i+1}-U_{i}\right)=\bar{A}\left(U_{i+1}-U_{i}\right)
$$

there exists $x, y \in \mathbb{R}^{2}$ such that $U_{i+1}-U_{i}=x r_{1}+y r_{2}$, and hence

$$
F^{\mathrm{Roe}}\left(U_{i+1}, U_{i}\right)=\frac{1}{2}\left(F\left(U_{i+1}\right)+F\left(U_{i}\right)-|u|\left(U_{i+1}-U_{i}\right)\right) .
$$

Thanks to this, it is clear that all the calculations made before for the Rusanov fluxes extend to this case. The only modification is that the numerical dissipation is now $\bar{u}=u$.

We get that $u_{i}=u$ and $P_{i}=P$ for any $i$ implies that $u_{i}^{n+1}=u$ for any $i$. In order to enforce the condition $P_{i}^{n+1}=P$, we need that

$$
\chi_{i}^{n+1}=\chi_{i}^{n}-\lambda\left[u_{i}^{n}\left(\chi_{i+1}^{n}-\chi_{i-1}^{n}\right)-|\bar{u}|\left(\chi_{i+1}^{n}-2 \chi_{i}^{n}+\chi_{i-1}^{n}\right)\right],
$$

where $\lambda=\frac{\Delta t}{2 \Delta x}$.

3.5.3. Extension with an exact Riemann solver. We follow the same procedure. The numerical fluxes are computed from the solution of the Riemann problem with an exact solver. Such a solver is described, for example, in Cocchi, Saurel, and Loraud [5] and Toro [34]. At a cell boundary, the exact Riemann solver provides the solution for $\rho^{*}, u^{*}, P^{*}, e^{*}, \alpha^{*}$, and $\beta^{*}$. We note $\lambda=\frac{\Delta x}{\Delta t}$ and we first examine the Godunov scheme applied to the mass conservation equation. We get

$$
\rho_{i}^{n+1}=\rho_{i}^{n}-\lambda\left[\left(\rho^{*} u^{*}\right)_{i+1 / 2}^{n}-\left(\rho^{*} u^{*}\right)_{i-1 / 2}^{n}\right] .
$$

We again assume $u_{i}^{n} \equiv u=u^{*}$, so

$$
\rho_{i}^{n+1}=\rho_{i}^{n}-\lambda u\left[\left(\rho^{*}\right)_{i+1 / 2}^{n}-\left(\rho^{*}\right)_{i-1 / 2}^{n}\right] .
$$

Now we develop the Godunov scheme for the momentum conservation equation:

$$
\rho u_{i}^{n+1}=\rho u_{i}^{n}-\lambda\left[\left(\rho^{*}\left(u^{*}\right)^{2}+P^{*}\right)_{i+1 / 2}^{n}-\left(\rho^{*}\left(u^{*}\right)^{2}+P^{*}\right)_{i-1 / 2}^{n}\right] .
$$


Combining (3.9) and (3.10), the equality of pressure $P_{i}^{n} \equiv P^{*}=P$, and velocity at time $t_{n}$, we again get $u_{i}^{n+1}=u_{i}^{n}$. Finally, we consider the equation for the total energy:

$$
\rho e_{i}^{n+1}=\rho e_{i}^{n}-\lambda\left[\left(\rho^{*} e^{*} u^{*}+P^{*} u^{*}\right)_{i+1 / 2}^{n}-\left(\rho^{*} e^{*} u^{*}+P^{*} u^{*}\right)_{i-1 / 2}^{n}\right] .
$$

Using previous equations and assumptions, we get

$$
\rho I_{i}^{n+1}=\rho I_{i}^{n}-\lambda u\left[\left(\rho^{*} I^{*}\right)_{i+1 / 2}^{n}-\left(\rho^{*} I^{*}\right)_{i-1 / 2}^{n}\right]
$$

With the help of $(3.1), \rho I=\alpha P+\beta$; then we get

$(\alpha P)_{i}^{n+1}+\beta_{i}^{n+1}=\left\{(\alpha P)_{i}^{n}-\lambda u P\left(\alpha_{i+1 / 2}^{* n}-\alpha_{i-1 / 2}^{* n}\right)\right\}+\left\{\beta_{i}^{n}-\lambda u\left(\beta_{i+1 / 2}^{* n}-\beta_{i-1 / 2}^{* n}\right)\right\}$.

Hence, we obtain $P_{i}^{n+1}=P$ under the condition

$$
\chi_{i}^{n+1}=\chi_{i}^{n}-\lambda u_{i}^{n}\left(\chi_{i+1 / 2}^{* n}-\chi_{i-1 / 2}^{* n}\right)
$$

where $\chi=(\alpha, \beta)^{T}$.

To summarize, when the original Godunov scheme is used our scheme is written

$$
\left\{\begin{array}{l}
U_{i}^{n+1}=U_{i}^{n}-\lambda\left(F_{i+1 / 2}^{*}-F_{i-1 / 2}^{*}\right) \\
\chi_{i}^{n+1}=\chi_{i}^{n}-\lambda u_{i}^{n}\left(\chi_{i+1 / 2}^{* n}-\chi_{i-1 / 2}^{* n}\right)
\end{array}\right.
$$

3.6. Extension to multidimensions. The solution in multidimensions can be obtained by applying the well-known principles of operator splitting. For example, the $2 \mathrm{D}$ operator can be approximated by a succession of $1 \mathrm{D}$ sweeps [32]

$$
U_{i}^{n+1}=\left(L_{x}^{\frac{\Delta t}{2}} L_{y}^{\Delta t} L_{x}^{\frac{\Delta t}{2}} U^{n}\right)_{i}
$$

where $L_{x}$ (resp., $L_{y}$ ) represents the $1 \mathrm{D}$ operator along $x$ direction (resp., $y$ ) as described previously. Some precautions must be taken in order to preserve equality of pressure and velocities during each sweep. Let us examine for example the $x$-sweep. The same remarks apply directly to the $y$-sweep. Equations to solve during the $x$ sweep are

$$
\frac{\partial U}{\partial t}+\frac{\partial F}{\partial x}=0
$$

with $U=(\rho, \rho u, \rho v, \rho e)^{T}$ and $F=\left(\rho u, \rho u^{2}+P, \rho u v, u(\rho e+P)\right)^{T}$, where $e=I+\frac{u^{2}+v^{2}}{2}$.

We consider the case of the Rusanov scheme, but other examples can be considered in the same spirit. Let us assume once more that the $x$-velocity is uniform while the $y$-component is not. The pressure is also assumed to be uniform. Following the same development as in section 3.2, (3.9) and (3.11) remain unchanged. The transverse momentum equation reads

$$
\rho v_{i}^{n+1}=\rho v_{i}^{n}-\lambda\left[u_{i}^{n}\left(\rho v_{i+1}^{n}-\rho v_{i-1}^{n}\right)-S_{i+1 / 2}\left(\rho v_{i+1}^{n}-\rho v_{i}^{n}\right)+S_{i-1 / 2}\left(\rho v_{i}^{n}-\rho_{i-1}^{n}\right)\right] .
$$


The total energy equation is

$$
\begin{aligned}
& (\alpha P)_{i}^{n+1}+\beta_{i}^{n+1}+\left(\rho \frac{v^{2}}{2}\right)_{i}^{n+1} \\
& \quad=(\alpha P)_{i}^{n}-\lambda\left[u_{i}^{n} P_{i}^{n}\left(\alpha_{i+1}^{n}-\alpha_{i-1}^{n}\right)-S_{i+1 / 2} P_{i}^{n}\left(\alpha_{i+1}^{n}-\alpha_{i}^{n}\right) S_{i-1 / 2} P_{i}^{n}\left(\alpha_{i}^{n}-\alpha_{i-1}^{n}\right)\right] \\
& \quad+\beta_{i}^{n}-\lambda\left[u_{i}^{n}\left(\beta_{i+1}^{n}-\beta_{i-1}^{n}\right)-S_{i+1 / 2}\left(\beta_{i+1}^{n}-\beta_{i}^{n}\right)+S_{i-1 / 2}\left(\beta_{i}^{n}-\beta_{i-1}^{n}\right)\right] \\
& \quad+\left(\rho \frac{v^{2}}{2}\right)_{i}^{n}-\lambda\left[u_{i}^{n}\left\{\left(\rho \frac{v^{2}}{2}\right)_{i+1}^{n}-\left(\rho \frac{v^{2}}{2}\right)_{i-1}^{n}\right\}-S_{i+1 / 2}\left\{\left(\rho \frac{v^{2}}{2}\right)_{i+1}^{n}-\left(\rho \frac{v^{2}}{2}\right)_{i}^{n}\right\}\right. \\
& \left.\quad+S_{i-1 / 2}\left\{\left(\rho \frac{v^{2}}{2}\right)_{i}^{n}-\left(\rho \frac{v^{2}}{2}\right)_{i-1}^{n}\right\}\right] .
\end{aligned}
$$

We see that to have $P_{i}^{n+1}=P_{i}^{n}$ we have to impose (3.12) on $\chi$ and (denoting $\left.K_{t}=\rho \frac{v^{2}}{2}\right)$

$$
K_{t_{i}}^{n+1}=K_{t_{i}}^{n}-\lambda\left[u_{i}^{n}\left(K_{t_{i+1}}^{n}-K_{t_{i-1}}^{n}\right)-S_{i+1 / 2}\left(K_{t_{i+1}}^{n}-K_{t_{i}}^{n}\right)+S_{i-1 / 2}\left(K_{t_{i}}^{n}-K_{t_{i-1}}^{n}\right)\right] .
$$

This is not surprising because it can be seen that even in the case of a perfect gas, when the initial condition corresponds to a slip line aligned with the mesh, the pressure starts to deviate from the initial value as well as the tangential and normal component of the velocity. In most cases, this effect is indeed very small. The effect of this default is not yet well understood.

It is possible to derive a scheme that will genuinely preserve the slip line (aligned with the mesh). The idea is to consider the system

$$
\begin{aligned}
& \frac{\partial U^{\prime}}{\partial t}+\frac{\partial F^{\prime}}{\partial x}=0, \\
& \frac{\partial v}{\partial t}+u \frac{\partial v}{\partial x}=0
\end{aligned}
$$

where $U^{\prime}=\left(\rho, \rho u, \rho e^{\prime}\right)^{T}$ and $F^{\prime}=\left(\rho u, \rho u^{2}+P, u\left(\rho e^{\prime}+P\right)\right)^{T}$ with $e^{\prime}=e-\frac{v^{2}}{2}$, that can be derived from the original Euler equations. Then applying once more the same principles, we can obtain a discretization which will be consistent with the preservation of slip lines. The difficulty, as it can easily be seen, is that (3.32) is valid only in the $x$-direction. For the $y$-direction, another value of $e^{\prime}$ (namely $e^{\prime}=e-\frac{u^{2}}{2}$ ) has to be considered. The synchronization of the $x$ and $y$ sweeps has to be done, with the consequence that strict conservation of the energy is lost. Due to the complexity of the coding, as well as the very weak effect of this subtle modification, we have considered the scheme based on (3.31) only.

Thus to summarize, for each time step and for each cell boundary, we start from $(\rho, \rho u, \rho v, \rho e, \alpha, \beta)$ at time $t_{n}$. We consider then the case of the $x$-sweep with a time step $\Delta t / 2$. We compute the tangential kinetic energy $K_{t}$ at time $t_{n}: K_{t}=\frac{\left(\rho^{n} v^{n}\right)^{2}}{2 \rho^{n}}$. We update $(\rho, \rho u, \rho v, \rho e)^{T}$ by the conservative scheme, $(\alpha, \beta)$ by $(3.13)$, and $K_{t}$ by (3.31). We deduce the internal energy by $(\rho I)^{n+1}=(\rho e)^{n+1}-K_{t}^{n+1}-\frac{\left(\rho^{n+1} u^{n+1}\right)^{2}}{2 \rho^{n+1}}$ and the pressure by $\alpha^{n+1} P^{n+1}+\beta^{n+1}=(\rho I)^{n+1}$. Then we drop the tangential kinetic energy $K_{t}^{n+1}$ and we start the same procedure on the $y$-sweep with the time step $\Delta t$, and so on. 

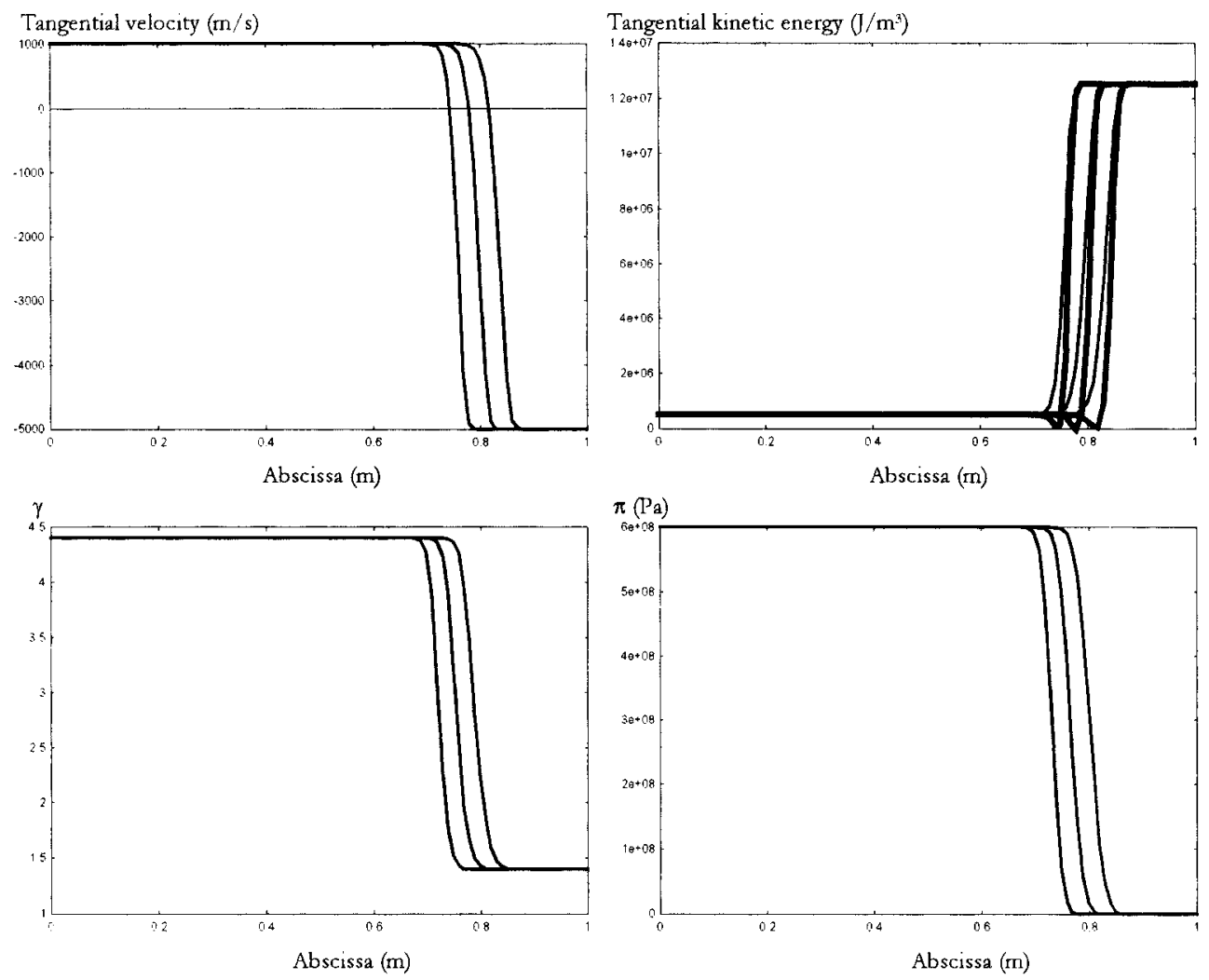

FIG. 2. Time evolution of the tangential velocity and kinetic energy and equation of state parameters.

If the tangential kinetic energy is not convected, the numerical errors become so large for very strong slip lines that the scheme blows up. This can be clearly seen in Figure 2 where the nonmonotonic behavior of the transversekinetic energy is responsible for the code blowup: (3.31) guaranties a monotone profile of $K_{t}$ under the CFL condition.

4. Results. All the results we present are obtained with the HLL-Riemann solver and the van Albada, van Leer, and Roberts [36] slope limiter, in the context of the second order variant of the method. We do not show the results for the Rusanov fluxes because this numerical flux is more diffuse. We have not used the Roe scheme because it is not always able to compute accurate solutions on the drastic conditions of the following test problems. The method using the exact Riemann solver is the most accurate and will be tested on the first test problem but not on multidimensional problems because of the computational cost.

In most cases we provide the density evolution. For special applications, even if the initial densities of the various fluids are very different, during the run the density of the two fluids may become close. The density contours are no longer characteristic of the interface location. This is why we have added to the system of equations an auxiliary equation for the evolution of the color function $f$. This color function is set to zero in one fluid initially and to one in the other fluid when we deal with two fluids 
only. This function is used for plots only. This equation may be written as

$$
\frac{\partial \rho f}{\partial t}+\nabla \cdot(\rho f \vec{u})=0
$$

and is solved by the conservative scheme. The numerical flux is obtained by upwinding in the direction indicated by the mass flux. The equation of state variables can also be used for representation of the interface, but their resolution by the nonconservative scheme is more dissipative than the conservative resolution of (4.1).

4.1. A two-phase shock tube. The method relies on the assumption of a uniform velocity and pressure flow in each mesh point. Here we test the capabilities of the method to work on a Riemann problem where all the variables (including the pressure) are strongly discontinuous. It consists of a tube filled in its left part by a liquid at high pressure and on the right by a gas at low pressure. On such a test case, standard shock capturing methods fail at the second time step (negative argument in the speed of sound computation). The initial data are

- Liquid: $\rho_{l}=1000 \mathrm{~kg} / \mathrm{m}^{3}, P_{l}=10^{9} \mathrm{~Pa}, u_{l}=0 \mathrm{~m} / \mathrm{s}, v_{l}=1000 \mathrm{~m} / \mathrm{s}, \gamma_{l}=4.4$, $\pi_{l}=6.10^{8} \mathrm{~Pa}$

- Gas: $\rho_{r}=50 \mathrm{~kg} / \mathrm{m}^{3}, P_{r}=10^{5} \mathrm{~Pa}, u_{r}=0 \mathrm{~m} / \mathrm{s}, v_{r}=-5000 \mathrm{~m} / \mathrm{s}, \gamma_{r}=1.4$, $\pi_{r}=0$.

The initial discontinuity is located at $x=0.7 \mathrm{~m}$, and the results are represented at $t=240 \mu \mathrm{s}$. The numerical solution is plotted in bold lines and the exact one in thin lines. Here, the mesh contains 1000 cells uniformly distributed to show mesh convergence. This is an indication that our method, although not strictly conservative, is able to compute discontinuities moving at the right speed. Figure 3 represents the excellent agreement between the two solutions. The upper graphs have been obtained with the method using the HLL solver, while the lower ones have be obtained with the scheme based on the exact Riemann solver. The HLL solver is used because of the stiffness of the problem. A solver which guarantees the positivity of $\rho$ and $P$ is mandatory, even in a simulation for an ideal gas, on these conditions. Some velocity oscillations are present on the tail of the rarefaction wave, and density oscillations are present at the contact discontinuity. The velocity oscillation is more visible when the HLL solver is used rather than the exact one. The density oscillation at the interface is present with both solvers. This type of oscillation is present even for single fluid calculations. Here, they are magnified because of the stiffness of the problem (density ratio of 20 and pressure ratio of $10^{4}$ ).

However, even with very few points (100) a rather good agreement between the exact and the numerical solutions can be obtained. In Figure 4, the velocity and pressure profiles are shown at three successive instants. Final curve is obtained after 90 time steps. Each curve is separated by 30 time steps. These results are obtained with the HLL solver. Similar results are obtained with the exact Riemann solver. Even if the method has been built for uniform pressure and velocity flows, it works on the present problem.

These 1D calculations have been obtained by the resolution of the $x$-split 2D system (3.30), corresponding to a sweep of the 2D alternate direction method. The tangential momentum equation has been solved. A discontinuous tangential velocity condition is assumed initially. A positive sliding velocity of $1000 \mathrm{~m} / \mathrm{s}$ is set into the left chamber, and a negative, nonsymmetric sliding velocity of $-5000 \mathrm{~m} / \mathrm{s}$ is set into the right chamber. Under these conditions, if the tangential kinetic energy is computed from the tangential velocity component, the method fails rapidly. It is why 

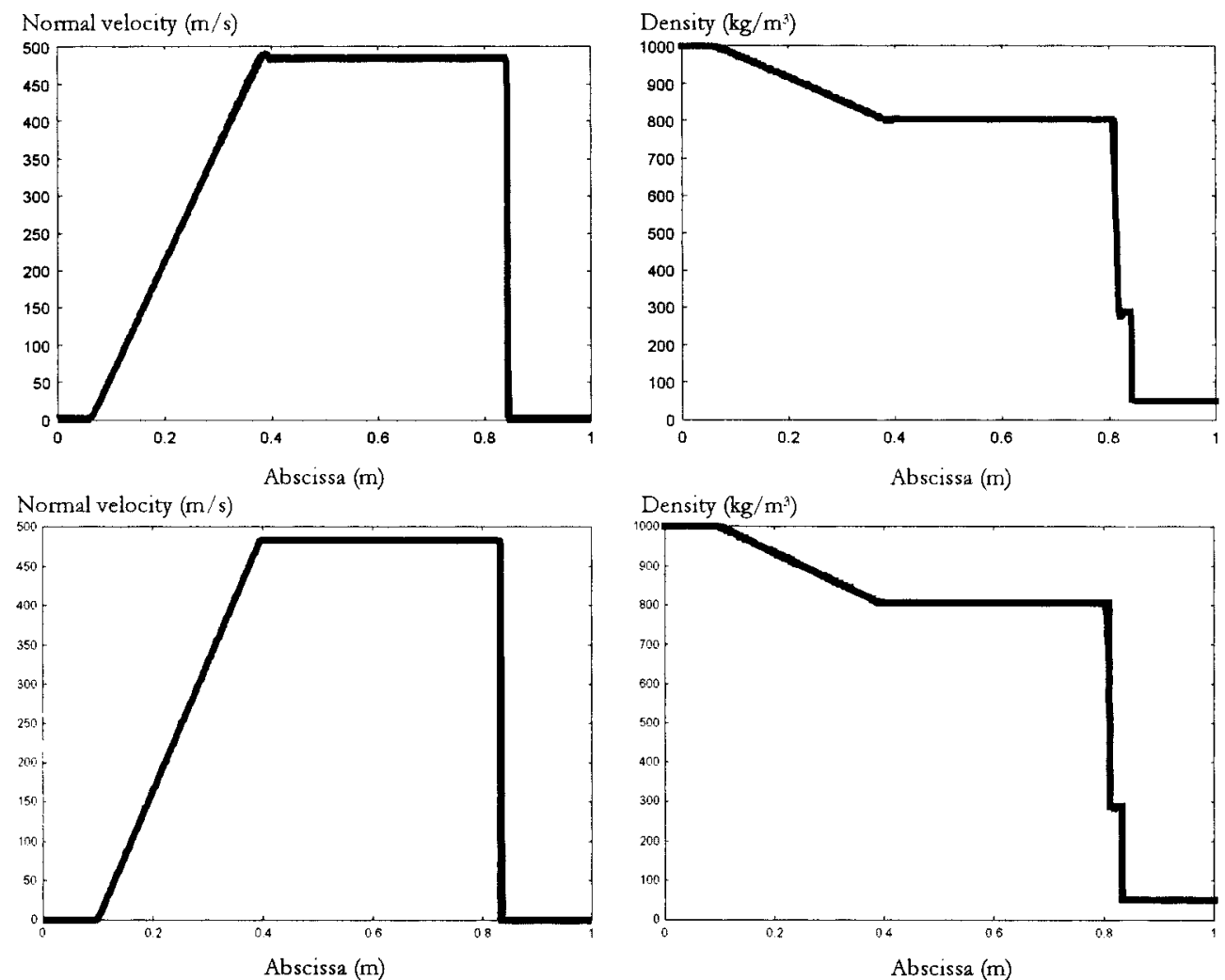

FIG. 3. Comparison of numerical schemes (bold lines) and exact solution (thin lines) for the liquid-gas shock tube. Upper graphs are obtained with the present second order method and HLL solver and the lower graphs with the present method and the exact Riemann solver.
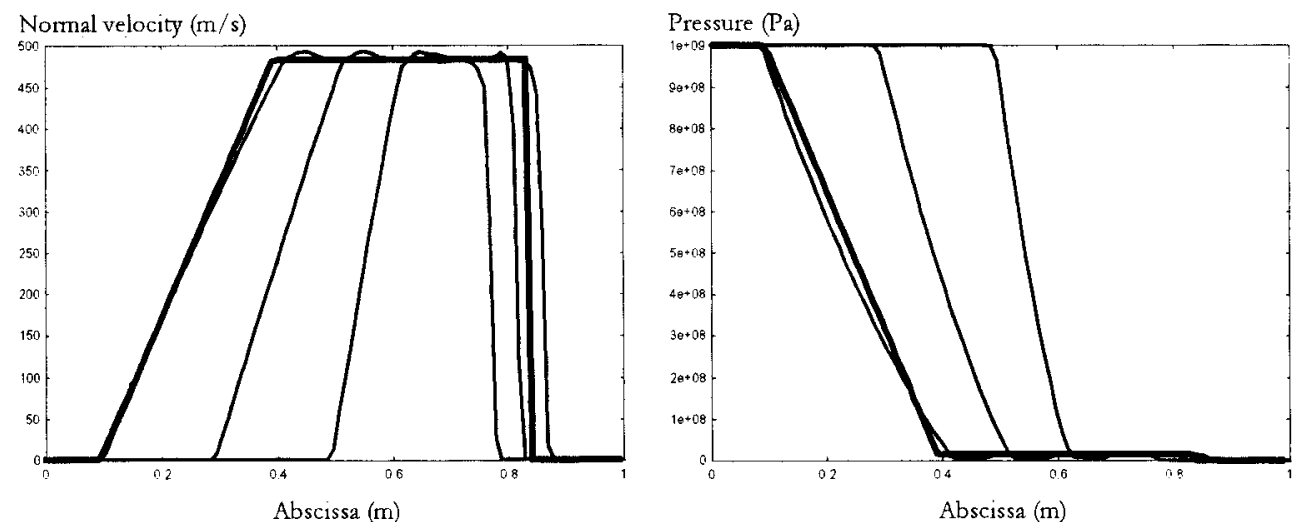

FIG. 4. Pressure and velocity unsteady evolutions. Exact solution is shown in bold lines at last time step.

the tangential kinetic energy must be computed from (3.31). We now look at this discontinuity evolution in Figure 2. The monotonic evolutions of $\gamma, \pi$, and $K_{t}$ are also provided. 
The solutions presented in Figures 4 and 2 are obtained with the HLL scheme. From the tangential velocity component, it is possible to calculate the tangential kinetic energy. Corresponding profiles are represented in bold lines in Figure 2. The thin lines correspond to the resolution of (3.31) and show the monotonic behavior of the tangential kinetic energy. One can notice that these results present strong differences. Kinetic energy computed from the tangential velocity component does not preserve pressure and velocity oscillations. Our method provides the correct results. It also can be noticed that the present methods provide monotonic behavior of all EOS parameters.

We now examine the behavior of the method on a 1D interaction of a strong rarefaction with a gas-liquid interface. This test case is interesting for pointing out that no density undershoots appear and that the pressure remains positive. We consider again a shock tube with three zones. The first chamber on the left side of the tube $(0<x<0.6 \mathrm{~m})$ is filled with liquid water under atmospheric conditions: $\rho=1000$ $\mathrm{kg} / \mathrm{m}^{3}, P=10^{5} \mathrm{~Pa}, u=0 \mathrm{~m} / \mathrm{s}$. The second one $(0.6 \mathrm{~m}<x<0.8 \mathrm{~m})$ is filled with air under atmospheric pressure: $\rho=50 \mathrm{~kg} / \mathrm{m}^{3}, P=10^{5} \mathrm{~Pa}, u=0 \mathrm{~m} / \mathrm{s}$. The last one $(0.8 \mathrm{~m}<x<1 \mathrm{~m})$ is filled with air at low pressure: $\rho=0.1 \mathrm{~kg} / \mathrm{m}^{3}, P=10^{3} \mathrm{~Pa}$, $u=0 \mathrm{~m} / \mathrm{s}$. This configuration results in a weak shock wave propagating on the right in the low-density air and a rarefaction wave propagating to the left and interacting with the gas-liquid interface. Corresponding results are shown in Figure 5.

These computations are made on a mesh of 100 cells. They clearly show that no density oscillation induce negative pressure.

4.2. Advection of a square gas bubble in uniform liquid flow. We now consider a very simple $2 \mathrm{D}$ test case. It consists of the advection of a square gas bubble in a liquid. Both phases are at the same pressure and velocity, so only translation of the gas bubble is observed. The initial data of this test problem are

- Liquid: $\rho_{l}=1000 \mathrm{~kg} / \mathrm{m}^{3}, P_{l}=10^{5} \mathrm{~Pa}, u_{l}=1000 \mathrm{~m} / \mathrm{s}, v_{l}=1000 \mathrm{~m} / \mathrm{s}$, $\gamma_{l}=4.4, \pi_{l}=610^{8} \mathrm{~Pa}$;

- Gas: $\rho_{g}=10 \mathrm{~kg} / \mathrm{m}^{3}, P_{g}=10^{5} \mathrm{~Pa}, u_{g}=1000 \mathrm{~m} / \mathrm{s}, v_{g}=1000 \mathrm{~m} / \mathrm{s}, \gamma_{g}=1.4$, $\pi_{g}=0$.

The computational domain is a 1-meter square with a $300 \times 300$ mesh, and the initial square bubble whose initial dimensions are $0.2 \mathrm{~m} \times 0.2 \mathrm{~m}(60 \times 60$ mesh points $)$ is located at the left bottom. Its center is located at $x=0.3 \mathrm{~m}$ and $y=0.3 \mathrm{~m}$. This simulation is performed with a Courant number of 0.8 . The results are represented at the initial time and after 600 time steps. Figure 6 represents density contours.

The numerical diffusion of the interface is clearly shown, but one can notice that the overall shape is preserved. It is possible to reduce this numerical diffusion by employing a "three waves" Riemann solver. This is not crucial for the following applications. The Roe and the exact Riemann solver contain three waves. They could be used for the present calculations. The velocity and pressure field are not represented but a cross cut of the corresponding variables at the last instant is shown on Figure 7. There are no pressure and velocity variations, contrary to what would be observed with a more classical solver.

4.3. Shock wave interaction with density discontinuities. We first test the capabilities of the method for the resolution of the interaction of a shock wave propagating in a liquid and interacting with a gas cylinder. It is a more difficult problem, both numerically and physically. The computational domain is 2 meters long and 1 meter high. It contains liquid at rest in atmospheric conditions. The gas is inside a cylinder whose center is located at $x=0.5$ meter and $y=0.5$ meter; its 

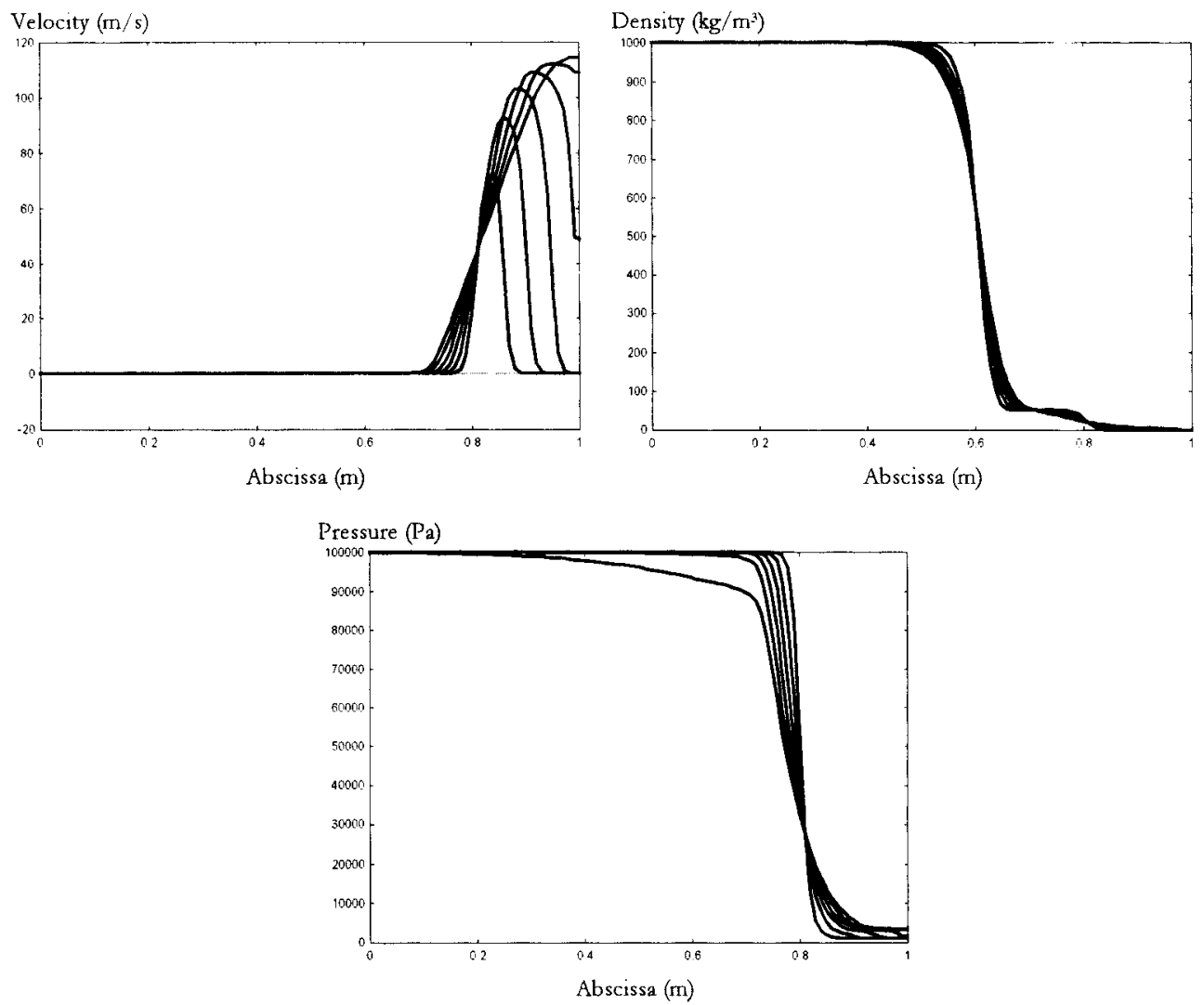

FIG. 5. Time evolution of the density, velocity, and pressure on the rarefaction tube problem. Each curve is separated by $0.25 \mathrm{~ms}$.

radius is 0.4 meter. A piston hits the left side at the velocity of $300 \mathrm{~m} / \mathrm{s}$ yielding a shock pressure of about $3.10^{9} \mathrm{~Pa}$. The top and bottom boundaries are solid walls.

The initial data of this test problem are

- Liquid: $\rho_{l}=1000 \mathrm{~kg} / \mathrm{m}^{3}, P_{l}=10^{5} \mathrm{~Pa}, u_{l}=0 \mathrm{~m} / \mathrm{s}, v_{l}=0 \mathrm{~m} / \mathrm{s}, \gamma_{l}=4.4$, $\pi_{l}=6.10^{8} \mathrm{~Pa}$

- Gas: $\rho_{g}=1 \mathrm{~kg} / \mathrm{m}^{3}, P_{g}=10^{5} \mathrm{~Pa}, u_{g}=0 \mathrm{~m} / \mathrm{s}, v_{g}=0 \mathrm{~m} / \mathrm{s}, \gamma_{g}=1.4, \pi_{g}=0$. The Courant number for the simulation is 0.8 and results are plotted every 500 time steps. The numerical simulation of such a problem has been done in the case of two gases governed by the ideal gas equation of state by Quirk and Karni [29] and Greenough and Jacobs [12]. The corresponding experiments were first conducted by Haas and Sturtevant [14]. The numerical study of a similar problem with water is given by Grove and Menikoff [13] and Ding and Gracewski [8]. The numerical method used in these two papers is very different from the one proposed here. Grove and Menikoff used a front tracking scheme and Ding and Gracewski, a Lagrangian scheme. The reported results of these two studies were for short duration after the shock wave interaction. The formation of the vortex pair described in the following study was not calculated: the distortions were too strong and surface topology was too complex.

Figure 8 represents the color function contours. Figure 8(a) represents the initial 


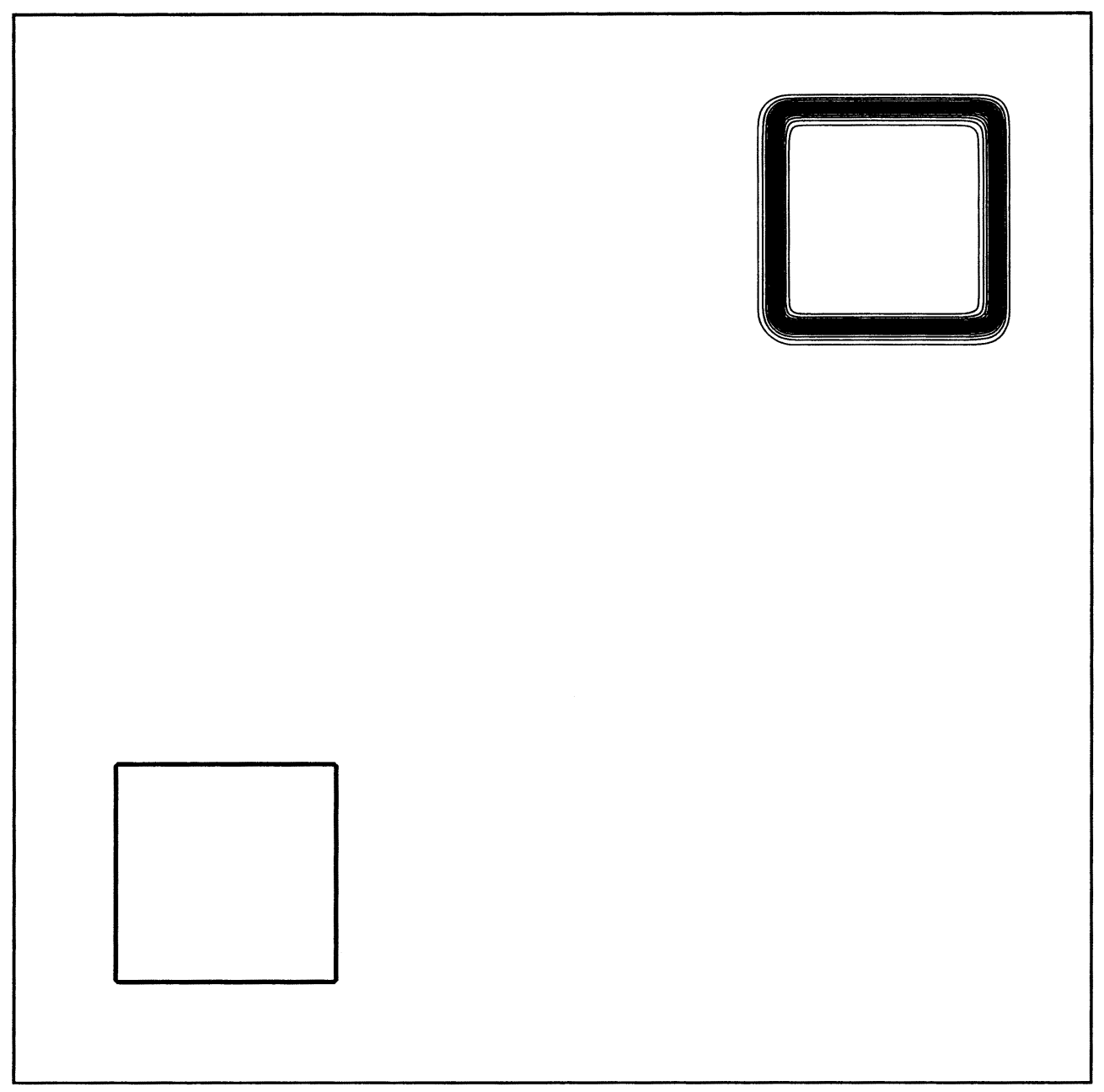

FIG. 6. Density contours for the advection of a square bubble.

condition. In Figure 8(b) there is a flattening of the bubble and a weak deformation of its original circular structure. From here to Figure 8(d), there is a strong deformation of the jet as it is inverted and transformed into a vortex pair. The first jet is created by the shock wave interaction with the interface along the symmetry axis. This interaction results in a transmitted weak shock wave in the gas and a refracted rarefaction wave facing to left. The combination of the two waves set into motion the interface. The rarefaction wave behaves as a nearly spherical wave. The acceleration is maximum near the symmetry line. This acceleration sets into motion the liquid on the left part of the bubble, creating a motion in the direction of this axis. Due to the symmetry condition, the flow deviates and forms a jet. An excellent description of this phenomena is given in Grove and Menikoff [13]. The creation of the vortex pair is explained in Greenough and Jacobs [12]. The vortex pair is a result of the jet deviation after its interaction with the opposite side of the bubble. In Figures 8(e) and (f), the flow evolves as a well-defined vortex pair. The corresponding density fields are represented in Figure 7. Due to the large density range of variation, the 

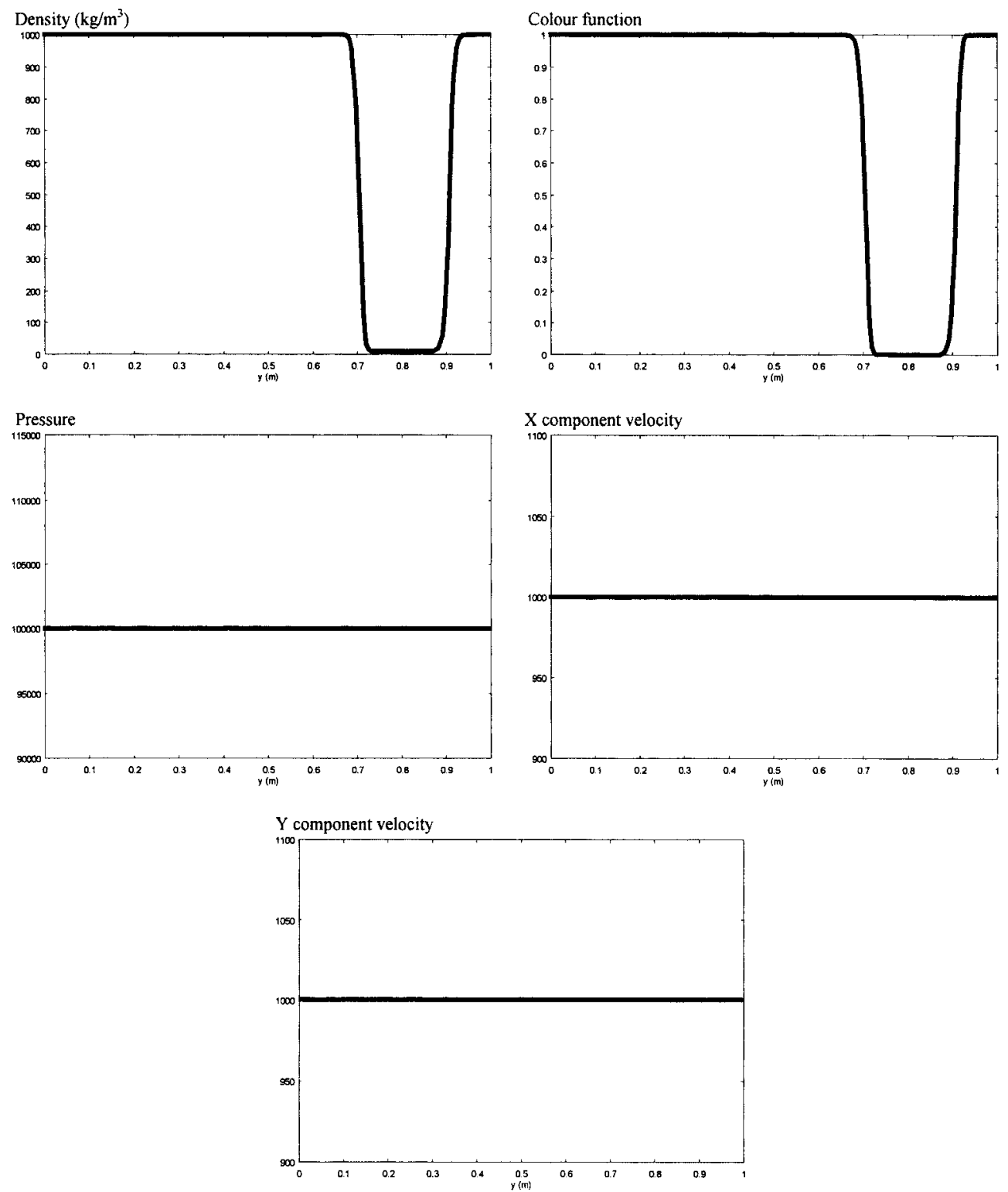

FIG. 7. Cross cut at $x=0.83$ meters for the square bubble advection test problem.

shock wave is not visible. Also, due to the strong compression of the gas inside the bubble, the contours of density are not representative of the interface position.

We show now the capability of the method for the resolution of the RichtmeyerMeshkov instabilities. It is another difficult problem of shock wave interaction with a density discontinuity. The computational domain is filled with two gases. The left part is filled with a light gas and the right part with a heavy one. They are separated by a curved interface. It is a portion of circle with center $x=1$ meter and $y=0.5$ meter and 0.6 meter radius. The physical domain is 4 meter long and 1 meter high. The mesh contains 500 cells along $x$-direction and 250 cells along $y$-direction. 
(a)

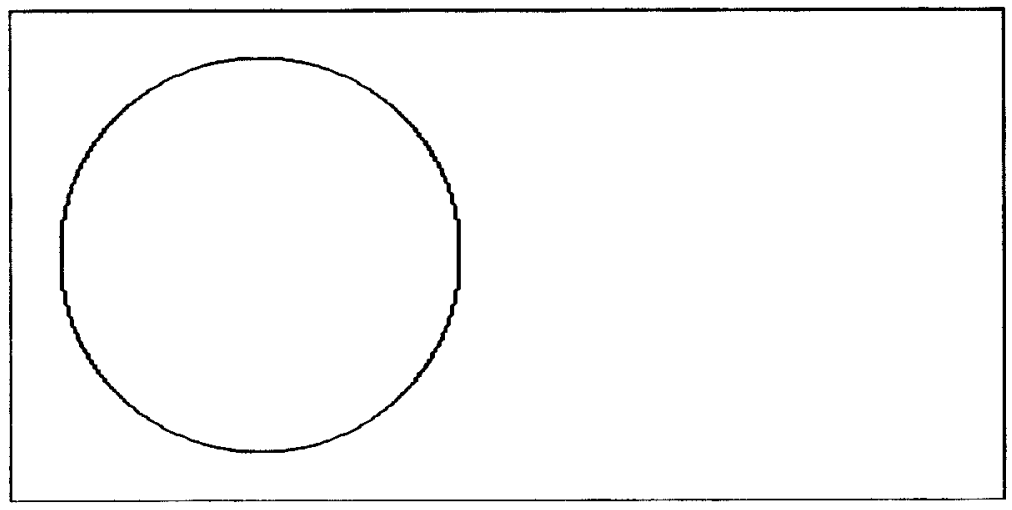

(b)

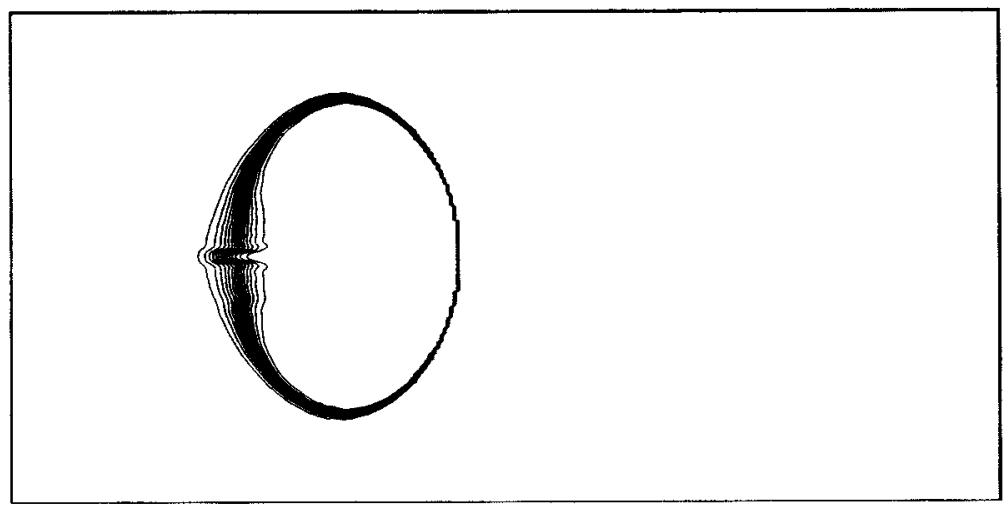

(c)

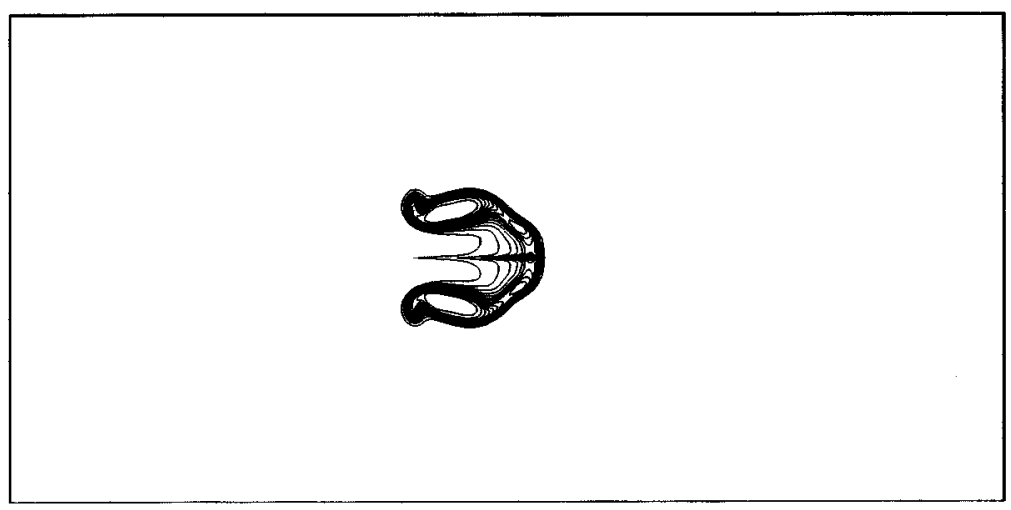

FIG. 8. Color function contours for the shock cylinder interaction problem.

The simulation needs 5000 time steps. The results are represented every 1000 steps. The Courant number is 0.8 . A piston hits the left side at the velocity of $500 \mathrm{~m} / \mathrm{s}$. The top and bottom boundaries are treated as solid walls. Initial data are

- Left: $\rho_{l}=1 \mathrm{~kg} / \mathrm{m}^{3}, P_{l}=10^{5} \mathrm{~Pa}, u_{l}=0 \mathrm{~m} / \mathrm{s}, v_{l}=0 \mathrm{~m} / \mathrm{s}, \gamma_{l}=1.4, \pi_{l}=0$; 
(d)

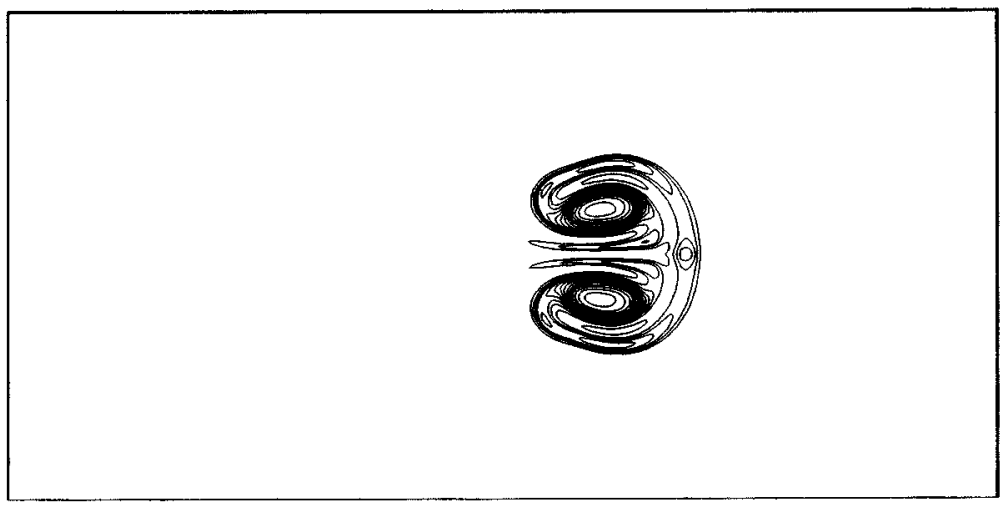

(e)

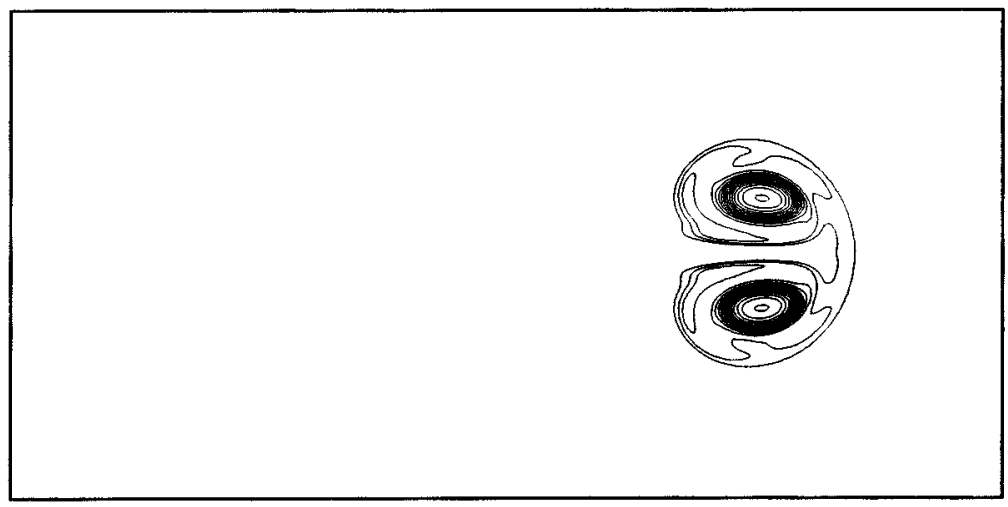

(f)

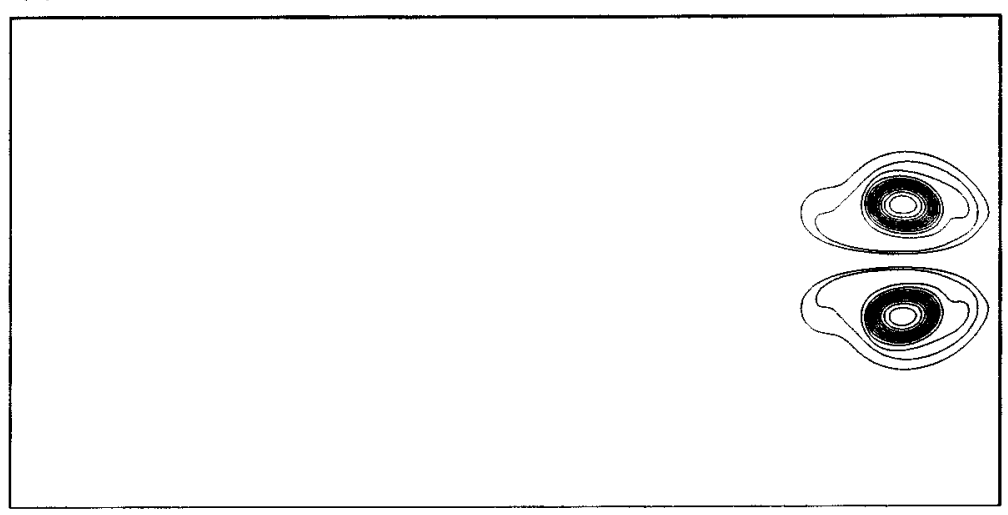

FIG. 6. (Continued).

- Right: $\rho_{r}=50 \mathrm{~kg} / \mathrm{m}^{3}, P_{r}=10^{5} \mathrm{~Pa}, u_{r}=0 \mathrm{~m} / \mathrm{s}, v_{r}=0 \mathrm{~m} / \mathrm{s}, \gamma_{r}=1.6$, $\pi_{r}=0$

Figure 8 represents the color function contours at six different times, starting from the initial condition on the top figure. The sequence of events occurring during this 

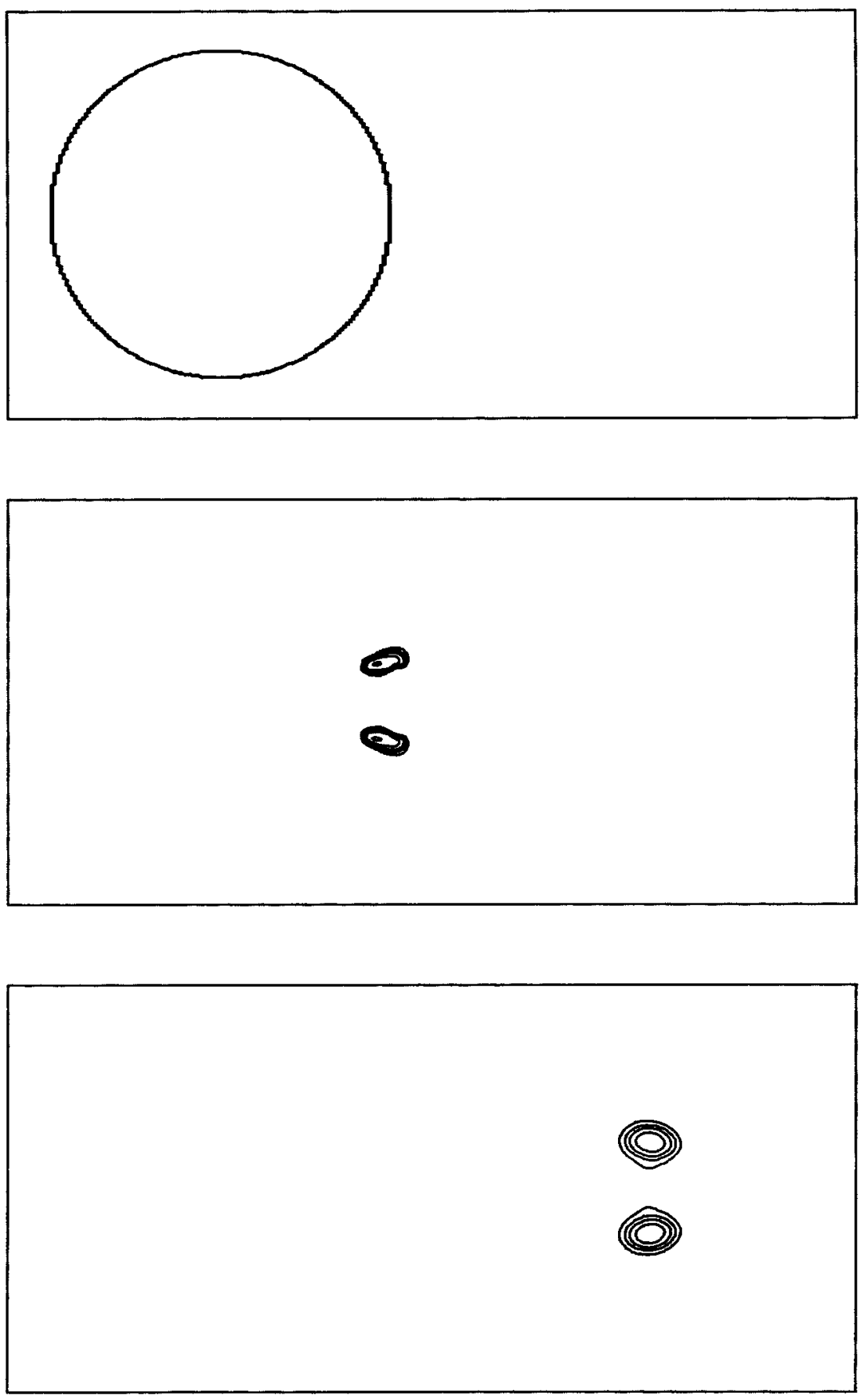

FIG. 7. Density contours for the shock cylinder interaction problem.

hydrodynamic phenomena is very complicated. For a description of these phenomena, the reader is referred to, for example, Jourdan and Houas [19].

4.4. 3D impact. We consider here the impact of a copper sphere on a liquid wall. The copper sphere has the initial velocity $u=2000 \mathrm{~m} / \mathrm{s}$ and $w=-1000 \mathrm{~m} / \mathrm{s}$. 

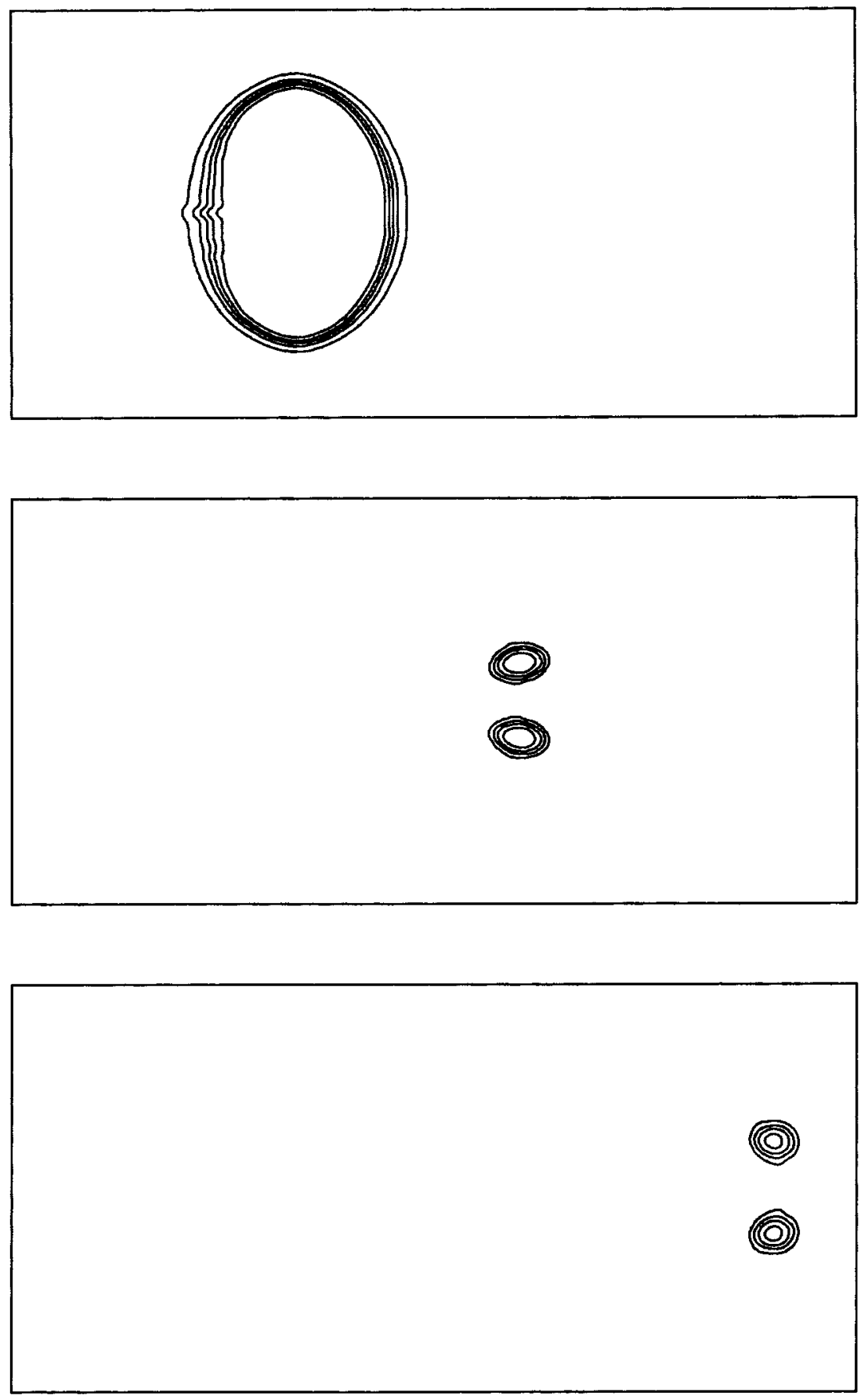

FIG. 7. (Continued).

Its center is located initially at $x c=0.2 \mathrm{~m}, y_{c}=0.5 \mathrm{~m}$, and $z_{c}=0.8 \mathrm{~m}$. Its radius is $0.15 \mathrm{~m}$. It hits the liquid (water) wall at rest, located between $x=0.36 \mathrm{~m}$ and $x=0.6 \mathrm{~m}$. The other part of the computational domain contains air at atmospheric conditions. The physical domain is 1 meter long, 1 meter high, and 

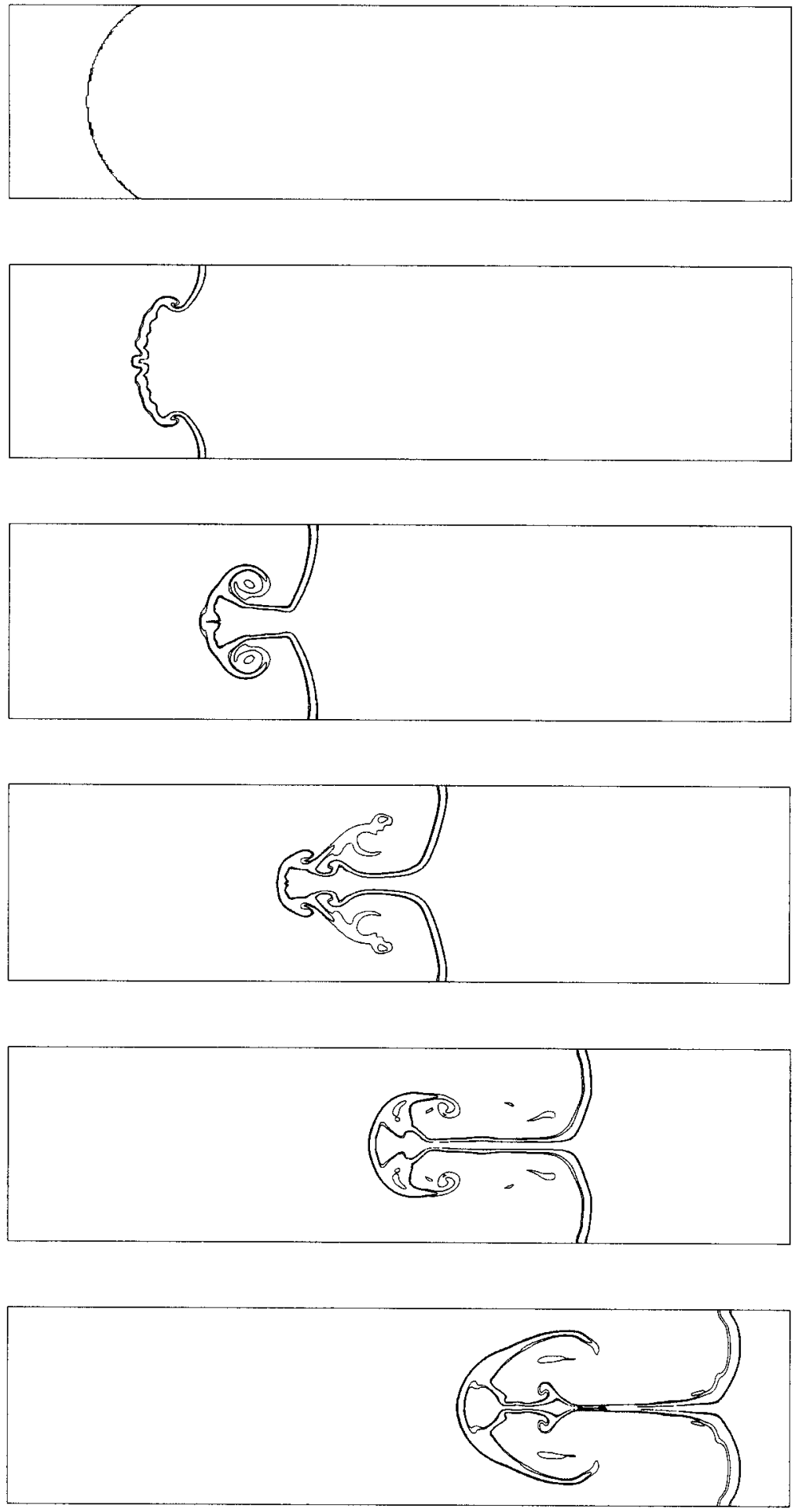

FIG. 8. Color function contours on the Richtmeyer-Meshkov instabilities test problem. 


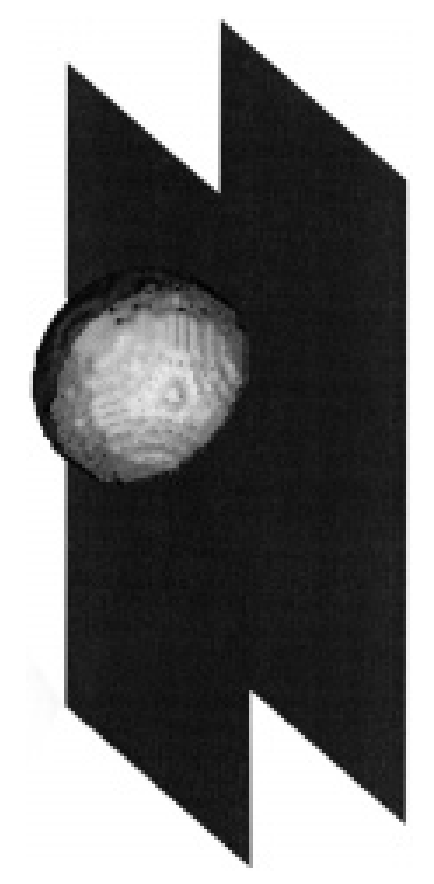

FIG. 9. Initial situation of the $3 D$ impact of a copper sphere on a liquid wall.

0.5 meter wide. The mesh contains 100 cells along $x$ - and $z$-directions and 50 cells along $y$-direction. The simulation is obtained with 400 time steps. The results are plotted every 80 steps. The Courant number is 0.8 . The parameters of each material are given in Table 3.1. Figure 9 shows the initial configuration. Figure 10 shows the beginning of the penetration of the copper sphere in the liquid wall. The copper sphere is weakly deformed and density gradients are visible. A shock wave is transmitted in the liquid and disturbs its surface. Figures 11 and 12 show the process continuation. The shock wave continues its propagation in the liquid and on its surface while the copper projectile is now well deformed and flattened. A surface discontinuity is created in the wake of the projectile and forms a crater. This crater increases in size in Figure 13 and the liquid layer begins to break up. In Figure 14 the breakup continues and the projectile exits the computational domain.

These figures show a sequence of events in reasonable agreement with what one can imagine. It shows that our method works without any difficulty in three dimensions on a three-material test case involving very high velocities and strong shock waves.

5. Conclusion. An extension of Abgrall's numerical method [2] has been described. It allows the numerical resolution of multifluid compressible flows with interfaces. It is a second order conservative TVD scheme combined with a second order nonconservative scheme. The efficiency of the subsequent method has been demonstrated on difficult test problems, such as shock bubble interaction, or Richtmeyer Meshkov instabilities. The method is accurate, robust, and very simple to code. 


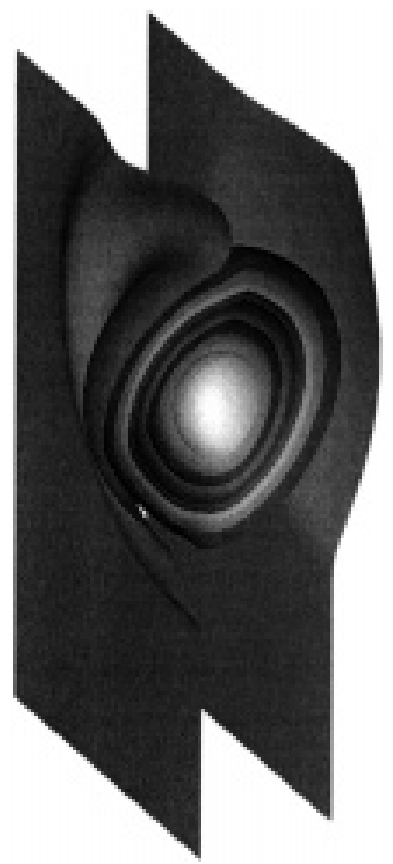

FIG. 10. Contours of the density at the beginning of penetration of the copper sphere into water.

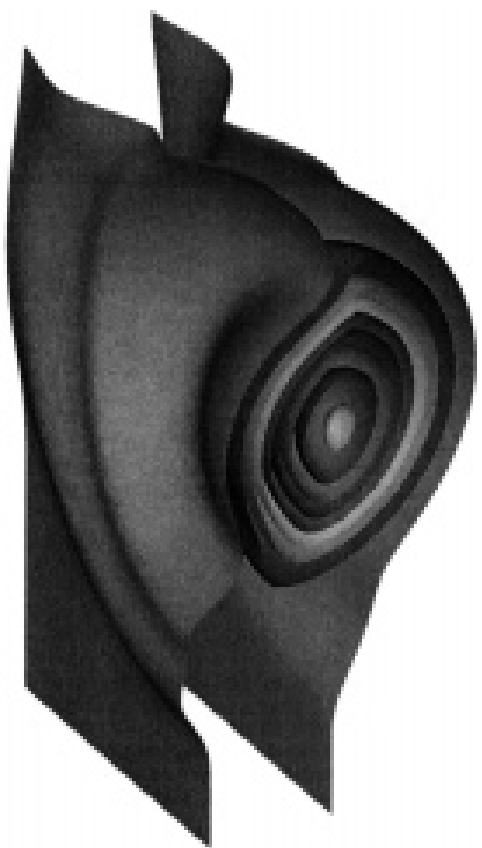

FIG. 11. Density contours after 160 time steps. 


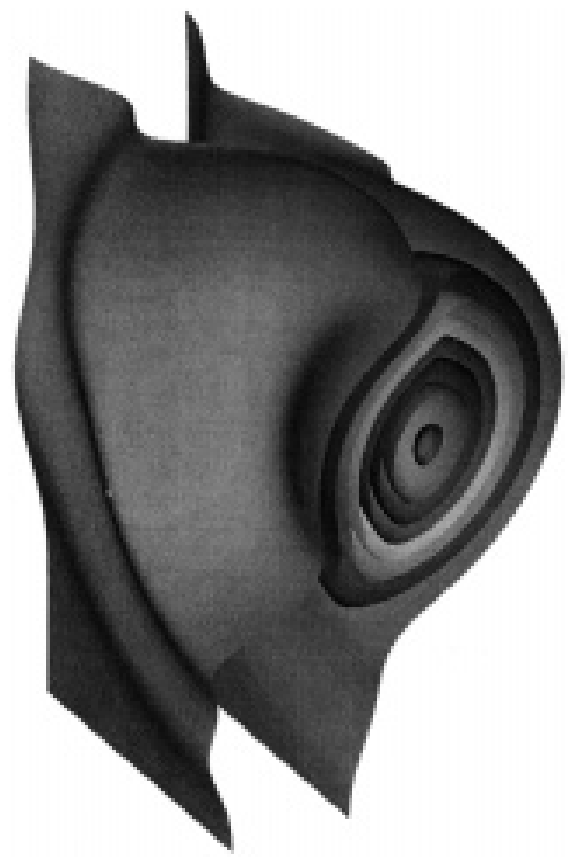

FIG. 12. Density contours after 240 time steps.

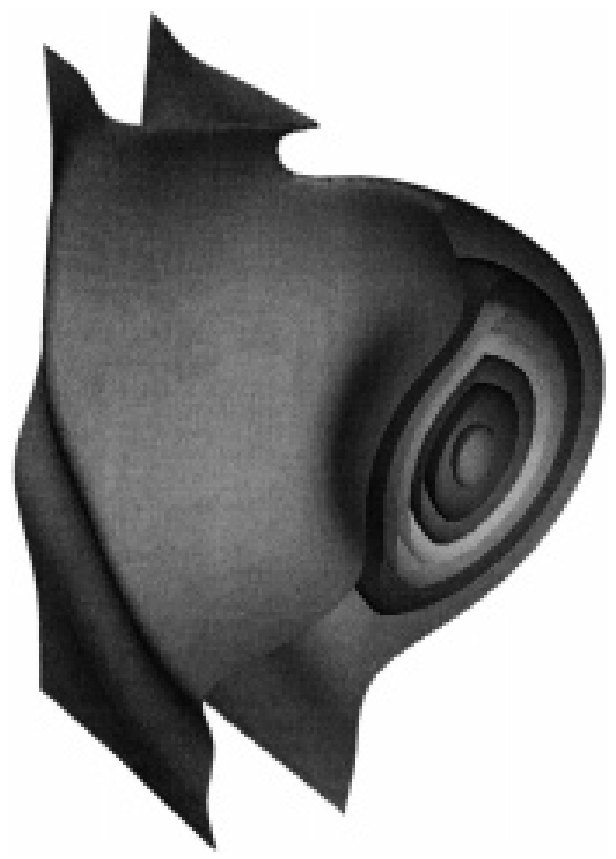

FIG. 13. Density contours after 320 time steps. 


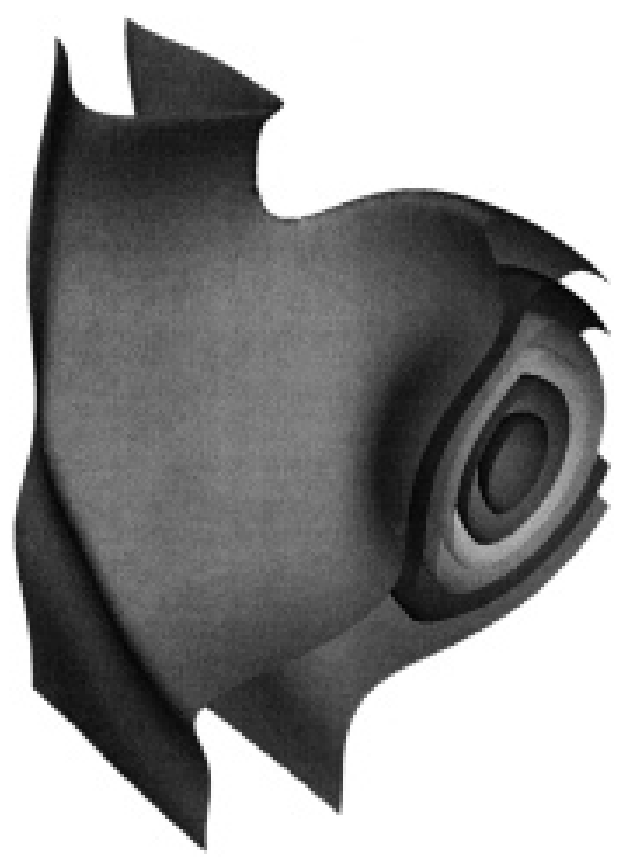

FIG. 14. Density contours after 400 time steps.

The 3D code is only 650 Fortran instructions. It requires only 40 Mbytes RAM for the $3 \mathrm{D}$ calculations presented herein $(500,000$ cells). Computational time is very reasonable: 3 hours for the shock-bubble interaction, 10 hours for the RichtmeyerMeshkov instabilities problem, and 5 hours for the 3D impact problem on a standard workstation.

The main limitation of the present method and our main perspective is related to the EOS. We plan to develop the algorithm for a more general EOS. We also examine the extension of this method for multidimensional problems on non-Cartesian grids.

Appendix A. Derivation of the Roe matrix. We start with some notation. If $f$ is any quantity (density, etc.) we denote $\bar{f}$ and $f$ by

$$
\bar{f}=\frac{\sqrt{\rho_{l}} f_{l}+\sqrt{\rho_{r}} f_{r}}{\sqrt{\rho_{l}}+\sqrt{\rho_{r}}}, \quad \underline{f}=\frac{\sqrt{\rho_{r}} f_{l}+\sqrt{\rho_{l}} f_{r}}{\sqrt{\rho_{l}}+\sqrt{\rho_{r}}}
$$

and we set $\Delta f=f_{l}-f_{r}$.

It is straightforward (but lengthy) to get $\bar{A}$ thanks to the following remarks (see [1]):

$$
\begin{array}{r}
\Delta(f g)=\bar{f} \Delta g+\underline{g} \Delta f, \\
\underline{\rho f}=\underline{\rho} \bar{f}, \\
\Delta\left(\frac{1}{f}\right)=-\frac{\Delta f}{f_{l} f_{r}}, \\
\overline{\frac{1}{f}}=\frac{1}{f_{l} f_{r}} \underline{f} .
\end{array}
$$


A systematic exploitation of the previous relations allows

$$
\widetilde{P}_{m}=\bar{\kappa} \bar{u}, \widetilde{P}_{E}=\bar{\kappa}, \widetilde{P}_{\rho Y_{i}}=\bar{\kappa} \bar{u}^{2}+\widetilde{\xi}_{i}
$$

where

$$
\kappa=\frac{1}{\sum_{i} \alpha_{i} Y_{i}} \text { and } \widetilde{\xi}_{i}=\widetilde{\kappa}_{\rho_{i} Y_{i}} \underline{\rho I}-\widetilde{\pi}_{\rho_{i} Y_{i}}
$$

with

$$
\begin{aligned}
\widetilde{\kappa}_{\rho_{i} Y_{i}} & =\frac{\underline{\rho \bar{Y}_{2}}\left(\alpha_{2}-\alpha_{1}\right)}{\left(\rho_{1} Y_{1} \alpha_{1}+\rho_{2} Y_{2} \alpha_{2}\right)_{l}\left(\rho_{1} Y_{1} \alpha_{1}+\rho_{2} Y_{2} \alpha_{2}\right)_{r}} \quad \text { and } \\
\tilde{\pi}_{\rho Y_{i}} & =\frac{-\underline{\rho}\left(\bar{Y}_{1} \beta_{1}+\bar{Y}_{2} \beta_{2}\right)+\underline{\rho}\left(\bar{Y}_{1} \alpha_{1}+\bar{Y}_{2} \alpha_{2}\right) \beta_{1}}{\left(\rho_{1} Y_{1} \alpha_{1}+\rho_{2} Y_{2} \alpha_{2}\right)_{l}\left(\rho_{1} Y_{1} \alpha_{1}+\rho_{2} Y_{2} \alpha_{2}\right)_{r}} .
\end{aligned}
$$

Similar relations are obtained for the partial derivatives related to the second fluid.

The relations (A.1), (A.2) are exploited as follows (for example). The first line of $\bar{A} \Delta U$ is

$$
\begin{aligned}
\bar{u} \bar{Y}_{2} \Delta\left(\rho Y_{1}\right)-\bar{u} \bar{Y}_{1} \Delta\left(\rho Y_{2}\right)+\bar{Y}_{1} \Delta(\rho u) & =\bar{u} \bar{Y}_{2}\left[\bar{Y}_{1} \Delta \rho+\underline{\rho} \Delta Y_{1}\right] \\
& -\bar{u} \bar{Y}_{1}\left[\bar{Y}_{2} \Delta \rho+\underline{\rho} \Delta Y_{2}\right]+\bar{Y}_{1} \Delta \rho u \\
& =\underline{\rho} \bar{u} \bar{Y}_{2} \Delta Y_{1}-\underline{\rho} \bar{u} \bar{Y}_{1} \Delta Y_{2}+\bar{Y}_{1} \Delta \rho u .
\end{aligned}
$$

Since $\Delta Y_{1}+\Delta Y_{2}=\Delta\left(Y_{1}+Y_{2}\right)=0$, we have

$$
\underline{\rho} \bar{u} \bar{Y}_{2} \Delta Y_{1}-\underline{\rho} \bar{u} \bar{Y}_{1} \Delta Y_{2} \quad=\underline{\rho} \bar{u}\left(\bar{Y}_{1}+\bar{Y}_{2}\right) \Delta Y_{1}+\bar{Y}_{1} \Delta \rho u=\underline{\rho u} \Delta Y_{1}+\bar{Y}_{1} \Delta \rho u=\Delta \rho u Y_{1},
$$

which is nothing more than the variation of $\rho u Y_{1}$.

\section{REFERENCES}

[1] R. Abgrall, Generalisation of the Roe scheme for the computation of mixture of perfect gases, Rech. Aérospat., 6 (1988), pp. 31-43 (English edition).

[2] R. AbGrall, How to prevent pressure oscillations in multicomponent flow calculations: A quasi conservative approach, J. Comput. Phys., 125 (1996), pp. 150-160.

[3] D. J. Benson, Comput. methods in Lagrangian and Eulerian hydrocodes, Comput. Methods Appl. Mech. Engrg., 99 (1992), pp. 235-394.

[4] J. P. Cocchi And R. Saurel, A Riemann problem based method for the resolution of compressible multimaterial flows, J. Comput. Phys., 137 (1997), pp. 265-298.

[5] J. P. Cocchi, R. Saurel, And J. C. Loraud, Treatment of interface problems with Godunovtype schemes, Shock Waves, 5 (1996), pp. 347-357.

[6] D. Chargy, R. Abgrall, L. Fezoui, and B. Larrouturou, Conservative numerical schemes for multicomponent inviscid flows, Rech. Aérospat., 2 (1992), pp. 61-79 (English version).

[7] S. F. Davis, Simplified second-order Godunov-type methods, SIAM J. Sci. Statist. Comput., 9 (1988), pp. $445-473$.

[8] Z. Ding, AND S. M. GRacewski, The behaviour of a gas cavity impacted by a weak or strong shock wave, J. Fluid Mech., 309 (1996), pp. 183-209.

[9] J. Glimm, J. W. Grove, X. L. Li, K.-M. Shyue, Y. Zeng, and Q. Zhang, Three-dimensional front tracking, SIAM J. Sci. Comput., 19 (1998), pp. 703-727.

[10] S. K. Godounov, A finite difference method for the numerical computation of discontinuous solutions of the equations of fluid dynamics, Math. Sb., 47 (1959), pp. 357-393.

[11] S. K. Godounov, A. Zabrodine, M. Ivanov, A. Kraiko, and G. Prokopov, Résolution numérique des problèmes multidimensionnels de la dynamique des gaz, Editions Mir, Moscow, 1979 
[12] J. A. Greenough And J. W. Jacobs, A numerical study of shock-acceleration of a diffuse helium cylinder, in Proceedings of the 5th International Workshop on Compressible Turbulent Mixing, Stony Brook, NY, R. Young, J. Glimm, and B. Boston, eds., World Scientific, River Edge, NJ, 1996.

[13] J. W. Grove and R. Menikoff, Anomalous reflexion of a shock wave at a fluid interface, J. Fluid Mech., 219 (1990), pp. 313-336.

[14] J. F. HAAS AND B. STURTEVAnt, Interaction of a weak shock wave with cylindrical and spherical gas inhomogeneities, J. Fluid Mech., 181 (1987), pp. 41-76.

[15] A. Harten and M. J. Hyman, Self adjusting grid methods for one-dimensional hyperbolic conservation laws, J. Comput. Phys., 50 (1983), pp. 235-269.

[16] A. Harten, P. D. Lax, AND B. VAn Leer, On upstream differencing and Godunov-type schemes for hyperbolic conservation laws, SIAM Rev., 25 (1983), pp. 35-61.

[17] F. Harlow and A. Amsden, Fluid Dynamics, Monograph LA-4700, Los Alamos National Laboratory, Los Alamos, NM, 1971.

[18] C. W. HiRT, AND B. D. Nichols, Volume of fluid method for the dynamics of free boundaries, J. Comput. Phys., 39 (1981), pp. 201-225.

[19] G. Jourdan and L. Houas, Proceedings of the Sixth International Workshop on the Physics of Compressible Turbulent Mixing, IUSTI, Marseille, France, 1997.

[20] S. KARNI, Multicomponent flow calculations by a consistent primitive algorithm, J. Comput. Phys., 112 (1994), pp. 31-43.

[21] S. KARNI, Hybrid multifluid algorithms, SIAM J. Sci. Comput., 17 (1996), pp. 1019-1039.

[22] P. D. Lax and P. Wendroff, Systems of conservation laws, Comm. Pure Appl. Math., (1960), pp. 217-237.

[23] R. J. Leveque And K. M. Shyue, Two-dimensional front tracking based on high resolution wave propagation methods, J. Comput. Phys., 123 (1996), pp. 354-368.

[24] D. K. MaO, A treatment of discontinuities for finite difference methods in two-dimensional case, J. Comput. Phys., 104 (1993), pp. 50, 235-269.

[25] S. P. Marsh, LASL Shock Hugoniot Data, University of California Press, Berkeley, CA, 1980.

[26] W. Mulder, S. Osher, And J. A. Sethian, Computing interface motion: The compressible Rayleigh-Taylor and Kelvin-Helmoltz instabilities, J. Comput. Phys., 100 (1992), p. 209.

[27] M. L. Norman AND K. H. A. WinkleR, $2 D$ Eulerian hydrodynamics with fluid interfaces selfgravity and rotation, in Astrophysical Radiation Hydrodynamics, K. H. A. Winkler and M. L. Norman, eds., pp. 187-221, D. Reidel, Boston, MA, 1986

[28] J. QUIRK, An alternative to unstructured grids for computing gas dynamic flow around arbitrarily complex two dimensional bodies, Comput. \& Fluids, 23 (1994), pp. 125-142.

[29] J. QuiRK AND S. KARni, On the Dynamics of a Shock-Bubble Interaction, NASA CR-194978; ICASE Rep. 94-75, NASA Langley Research Center, Langley, VA, 1994.

[30] P. L. Roe, Approximate Riemann solvers, parameter vectors and difference schemes, J. Comput. Phys., 43 (1981), pp. 357-372.

[31] V. V. Rusanov, Calculation of interaction of non-steady shock waves with obstacles, J. Comput. Math. Phys. USSR, 1 (1961), pp. 267-279.

[32] G. STRAnG, On the construction and comparison of difference schemes, SIAM J. Numer. Anal., 5 (1968), pp. 506-517.

[33] E. F. Toro, Some IVPs for which conservative methods fail miserably, in Proceedings of the 6th International Symposium on Comput. Fluid Dynamics, Lake Tahoe, CA, 1995.

[34] E. F. Toro, Riemann Solvers and Numerical Methods for Fluid Dynamics, Springer-Verlag, Berlin, 1997.

[35] B. VAN LEER, Toward the ultimate conservative difference scheme: V. A second order sequel to Godunov's method, J. Comput. Phys., 32 (1979), p. 101.

[36] G. D. van Albada, B. van Leer, and W. W. Roberts A comparative study of computational methods in cosmic gas dynamics, Astronom. Astrophys., 108 (1982), pp. 76-84.

[37] K. M. Shyue, An efficient shock-capturing algorithm for compressible multicomponent methods, J. Comput. Phys., 142 (1998), pp. 208-242.

[38] R. Young, J. Glimm, And B. Boston, Proceedings of the 5th International Workshop on Compressible Turbulent Mixing, World Scientific, River Edge, NJ, 1995.

[39] D. L. Youngs, Time dependent multi-material flow with large fluid distortion, in Numerical Methods for Fluid Dynamics, K. W. Morton and M. J. Baines, eds., Academic Press, London, New York, 1982. 